\title{
Scalable Pattern Sharing on Event Streams*
}

\author{
Medhabi Ray \\ Worcester Polytechnic Institute \\ 100 Institute Road \\ Worcester, MA 01609 \\ medhabi@cs.wpi.edu
}

\author{
Chuan Lei \\ Worcester Polytechnic Institute \\ 100 Institute Road \\ Worcester, MA 01609 \\ chuanlei@cs.wpi.edu
}

\author{
Elke A. Rundensteiner \\ Worcester Polytechnic Institute \\ 100 Institute Road \\ Worcester, MA 01609 \\ rundenst@cs.wpi.edu
}

\begin{abstract}
Complex Event Processing (CEP) has emerged as a technology of choice for high performance event analytics in time-critical decisionmaking applications. Yet it is becoming increasingly difficult to support high-performance event processing due to the rising number and complexity of event pattern queries and the increasingly high velocity of event streams. In this work we design the SPASS framework that successfully tackles these demanding CEP workloads. Our SPASS optimizer identifies opportunities for effective shared processing among CEP queries by leveraging time-based event correlations among queries. The problem of pattern sharing is shown to be NP-hard by reducing the Minimum Substring Cover problem to our CEP pattern sharing problem. The SPASS optimizer is designed that finds a shared pattern plan in polynomial-time covering all sequence patterns while still guaranteeing an optimality bound. To execute this shared pattern plan, the SPASS runtime employs stream transactions that assure concurrent shared maintenance and re-use of sub-patterns across queries. Our experimental study confirms that the SPASS framework achieves over 16 fold performance improvement for a wide range of experiments compared to the state-of-the-art solution.
\end{abstract}

\section{Keywords}

Complex Event Processing; Sharing; Sequence Pattern

\section{INTRODUCTION}

Motivation. Major businesses from fraud detection, online retail to health-care monitoring employ Complex Event Processing (CEP) technologies $[6,29,42]$ to detect event patterns such as time-ordered or unordered sequence of events, along with the nonexistence of events and other patterns, over high velocity event streams. Major advances have been made on the development of solutions for efficiently detecting event patterns in near real-time [6, $8,27,29,42]$. With the explosion in the number and type of

\footnotetext{
${ }^{*}$ This work was done when Medhabi Ray and Chuan Lei were students at Worcester Polytechnic Institute. NSF grants IIS-1018443 and 0917017 support this project.
}

Permission to make digital or hard copies of all or part of this work for personal or classroom use is granted without fee provided that copies are not made or distributed for profit or commercial advantage and that copies bear this notice and the full citation on the first page. Copyrights for components of this work owned by others than ACM must be honored. Abstracting with credit is permitted. To copy otherwise, or republish, to post on servers or to redistribute to lists, requires prior specific permission and/or a fee. Request permissions from permissions@ acm.org.

SIGMOD'16, June 26-July 01, 2016, San Francisco, CA, USA

(c) 2016 ACM. ISBN 978-1-4503-3531-7/16/06 . . $\$ 15.00$

DOI: http://dx.doi.org/10.1145/2882903.2882947 such event analytics queries, these systems face workloads that often involve hundreds or even thousands of real-time event pattern queries [27]. Pattern queries in these workloads can have subpatterns across numerous patterns that are concurrently executed in a shared manner. This points at a tremendous opportunity for scaling these compute intensive workloads.

However most existing CEP engines [6, 29, 42] process pattern matching queries sequentially without sharing nor maximizing concurrency among queries. With very high loads, such independent processing leads to repeated and thus wasted computations, exacerbating the problem of overload. This may cause a loss in the availability of service during peak hours, performance bottlenecks and potentially huge monetary loss for the organization due to not delivering results on time.

The running example shown in Figure 1 highlights the unique sharing opportunities available in a workload of pattern queries on a car location event stream similar to the Linear Road Benchmark data [2] for real-time traffic monitoring.

Motivating Example - Real-time Traffic Monitoring. The application monitors the motor vehicle expressways of a large metropolitan area. Monitoring movements of cars under various circumstances could be used for future route recommendations, toll adjustments and re-routing along with other intelligent traffic services. In this example, Carloc represents a stream of car location reports. The application sends out warnings to cars about possible delays due to constructions or accidents in a certain route. The first pattern $P_{1}$ monitors cars that first pass toll $A$, then pass a construction site and finally take the next exit. $P_{2}$ searches for traffic patterns where cars take an exit after seeing an accident while $P_{3}$ monitors cars that pass by a parked emergency vehicle and then take an exit.

\begin{tabular}{|c|c|}
\hline $\begin{array}{l}P_{1}: \text { PATTERN SEQ (CL (CL.pos = tollA), } \\
\text { CL1 (CL1.pos = WorkZone \& CL1.id = CL.id), } \\
\text { CL2 (CL2.pos = exitA \& CL.id = CL2.id)) } \\
\text { WITHIN } 30 \text { min on STREAM Carloc }\end{array}$ & $\begin{array}{l}\text { P2: PATTERN SEQ (CL (CL.pos = tollA), } \\
\text { Accident (Accident.pos }=\text { toll } A+5 \text { miles), } \\
\text { CL1 (CL1.pos = exitA \& CL.id }=C L 1 . i d) \text { ) } \\
\text { WITHIN } 30 \text { min on STREAM Carloc }\end{array}$ \\
\hline
\end{tabular}

P3: PATTERN SEQ (CL (CL.pos = tollA), CL1 (CL1. pos = CL. pos \& CL1. type = EV \& CL1. state $=$ Parked), CL2 (CL2.pos = exitA \& CL.id = CL2.id)) WITHIN 30 min on STREAM Carloc

\section{Figure 1: Pattern Query Workload}

The above queries are all monitoring the traffic around a particular toll booth (toll $A)$ and contain similar sub-patterns $S E Q$ $(C L(C L . p o s=$ toll $A), C L 2(C L 2 . p o s=$ exit $A \& C L . i d=$ $C L 2 . i d)$ ). Hence intermediate results could potentially be shared when processing these pattern queries, thus saving computational resources and achieving speed-up. Numerous event analytics applications from online advertising, click-stream analysis, to social 
networking services exhibit identical characteristics, namely, huge workloads composed of similar sequence pattern queries. The opportunity to share computations across such patterns is widespread.

Challenges. To design an effective infrastructure for scalable shared processing of a workload of sequence patterns, we must develop technologies to (1) first identify an effective pattern sharing plan, and (2) then to execute the shared pattern plan in an efficient concurrent manner.

- Benefit Estimation for Pattern Sharing. To identify sharable sub-patterns that minimize CPU costs, we need to establish an effective model to estimate the computational savings obtainable from sharing a given sub-pattern. One important observation here is that sharing a sequence pattern does not always result in true performance benefits. In our running example above, the events of witnessing a work zone in $P_{1}$ and witnessing an accident in $P_{2}$ are unlikely to co-occur. Thus the sharing of sub-patterns between $P_{1}$ and $P_{2}$ may even cause more harm than good by incurring unnecessary concurrency control overhead. Thus we propose to devise a lightweight yet effective method for estimating these time-sensitive co-occurrence properties of event streams to accurately capture the benefit of sharing sub-patterns of event pattern queries.

- Intractable Search Space of Pattern Sharing Optimization. Finding a set of sub-patterns to share among event patterns that minimizes the overall computation costs across the entire workload requires enumerating all possible combinations of sub-patterns. Similar to the view selection problem [30,37], i.e., choosing a set of views to materialize over a database such that the cost of evaluating a given query workload is minimized, identifying an optimal set of sub-patterns to share is prohibitively expensive due to its exponential search space [35]. This necessitates the design of practical yet effective strategies for finding a logical pattern sharing plan.

- Concurrent Shared Pattern Execution. Once a promising solution for sharing sub-patterns has been identified, the workload must be transformed into a shared runtime plan that can be executed in a concurrent manner. This requires a run-time infrastructure that correctly handles shared sub-pattern matches leveraging concurrent execution on a modern architecture. Shared sub-pattern matches must be maintained correctly so as to enable concurrent accesses by other queries involved in the sharing process.

State-of-the-Art. Prior work in CEP has mainly focused on the design of execution strategies for efficient event pattern processing $[6,8,26,42]$. These techniques adopt Nondeterministic Finite Automaton (NFA) execution strategy to process a single pattern at a time. Hence they do not concern themselves with exploiting sharing opportunities across the workload of pattern queries. While merging NFAs has been attempted in the context of XMLfiltering $[13,14]$, it is limited to prefix-matching. On the other hand, SPASS supports sharing of any common sub-patterns not necessarily limited to prefix or suffix patterns. There have been some efforts to execute event pattern matching on a distributed architecture [5, 36]. However, these works fail to provide any guarantee to produce a globally optimal plan for multiple pattern sharing.

In multiple query optimization (MQO), much work has been accomplished for static relational databases $[9,11,19]$. Prior work focused on view materialization for shared results across multiple queries [11] and on sharing similar sub-expressions to avoid recomputation costs [9]. However, these prior techniques cannot be directly applied to our continuous pattern sharing problem because (1) our data is continuously under flux, and (2) the data-driven approach of CEP may trigger the pattern matching process to be spawned in various orders based on the arrival of events - in contrast to the traditional static processing frameworks where the execution is fully orchestrated.
There has also been some work on MQO for continuous queries over data streams $[12,28]$. These works have focused on deciding at what level of data granularity (i.e., tuple, pane, or full window) to share a sub-expression across queries [28]. However without considering properties specific to pattern queries such as inter-query event correlations, optimization opportunities will be missed. This is now the focus of our work.

Proposed Solution. In this work we propose SPASS (독able

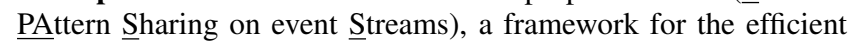
detection of event sequences in data streams via shared concurrent pattern execution. The SPASS optimizer takes as input a set of pattern queries. It estimates the benefit of sharing the computation of sub-patterns based on the time-ordering across events and the inter-query event correlation hidden in the event streams. We prove that our problem of finding an optimal pattern sharing plan is equivalent to the NP-hard Minimum Substring Cover (MSC) problem [21]. The approximate solution to the MSC problem with practical bounds on optimality [21] can now be applied to solve our problem. The resulting near-optimal shared pattern plan is then executed in a concurrent manner. For effective concurrent execution paradigm, we extend the continuous sliding views technology [34], initially designed for a single pattern query, to synchronously cache and reuse intermediate results of shared sub-patterns across multiple patterns. Lastly we introduce a stream transaction manager that controls access to these shared sliding views to achieve efficient yet correct insertion, deletion, and reuse of the sub-pattern matches.

Contributions. The contributions of this work include:

1. We establish a cost model for estimating the benefit of sharing sub-patterns by exploiting both the overlapping event ordering among pattern queries and inter-query event correlation hidden in event streams (Sec. 3).

2. We show that our problem of optimizing a workload of sequence pattern queries to minimize computation costs is equivalent to the well-known NP-hard problem, called Minimum Substring Cover [21]. This leads us to a polynomial-time solution to the problem of sequence pattern sharing, yet with proven acceptable bounds on optimality [21] (Sec. 3).

3. We propose an effective runtime infrastructure that facilitates shared processing of common sub-patterns across multiple queries by using the shared continuous sliding views technology. A stream transaction manager is introduced to guarantee synchronous access to these shared views for concurrent pattern executions (Sec. 4).

4. Our extensive experiments on real world event streams illustrate that our proposed SPASS approach achieves over 16 fold performance improvement on average for a wide range of scenarios over the state-of-the-art non-sharing and sharing (i.e., prefix- and suffix-based) approaches $[6,14,42]$ (Sec. 5).

\section{PRELIMINARIES}

\subsection{Event Processing Basics}

An event instance is defined as an occurrence of interest denoted by a lower-case letter (e.g., ' $e$ '). The time of occurrence of an event instance $e_{i}$ is given by a timestamp (referred to as $e_{i} . t s$ ). An event type $E$ of an instance $e_{i}$ denoted by $e_{i}$.type describes the set of attributes associated with $e_{i}$. A specific attribute of the event instance $e_{i}$ is referred to with $e_{i}$.attr. Predicates of the form $p_{i}\left(e_{i} . a t t r\right)$ can be specified on event instances. An event stream is an unbounded set of event instances $e_{i}$ that arrive in order of their occurrence time. The timestamp of the last event instance that has arrived into the system is referred to as $t s_{\text {curr }}$.

Query Specification. We adopt a pattern query language commonly used in the literature [27, 42] to specify event pattern queries. 
The example patterns in Figure 1 conform to the above query specification. We will explain each of these clauses below. The PATTERN clause denotes the event operator to be matched against the event stream specified in the FROM clause. In our SPASS framework, we support the common pattern operators, including $S E Q$, $A N D, O R$ and NOT [27, 42].

A SEQ operator outputs a event pattern match when a set of events conform to the order in timestamps and predicates specified in the operator within a given window.

$$
\begin{aligned}
& S E Q\left(E_{1}: p_{1}\left(E_{1} . \text { attr }\right), \ldots, E_{n}: p_{n}\left(E_{n} . \text { attr }\right)\right) \\
& =\left\{\left\langle e_{1}, \ldots, e_{n}\right\rangle \mid t s_{\text {curr }}-\text { Window }<e_{1} . t s<\ldots<e_{n} . t s\right. \\
& \left.<t s_{\text {curr }} \wedge\left(e_{i} . t y p e=E_{i}\right) \wedge p_{i}\left(e_{j} . \text { attr }\right)=\text { true }\right\}
\end{aligned}
$$

An AND operator outputs an event pattern match when a set of events occur within a certain time window in any order.

$$
\begin{aligned}
& A N D\left(E_{1}: p_{1}\left(E_{1} . \text { attr }\right), \ldots, E_{n}: p_{n}\left(E_{n} . a t t r\right)\right) \\
& =\left\{\left\langle e_{1}, \ldots, e_{n}\right\rangle \mid \forall e_{i}(i \in 1, \ldots n)\left(t s_{\text {curr }}-\text { Window }<e_{i} . t s\right.\right. \\
& \left.\left.\leq t s_{\text {curr }}\right) \wedge\left(e_{i} . \text { type }=E_{i}\right) \wedge p_{i}\left(e_{i} . \text { attr }\right)=\text { true }\right\}
\end{aligned}
$$

An OR operator denotes a union of a set of alternate event types. It produces an output when it finds a match for any one of the specified event types.

$$
\begin{aligned}
& \text { OR }\left(E_{1}: p_{1}\left(E_{1} . a t t r\right), E_{2}: p_{2}\left(E_{2} . a t t r\right), \ldots, E_{n}:: p_{n}\left(E_{n} . a t t r\right)\right) \\
& =\left\{\left\langlee_{1}: p_{1}\left(e_{1} . a t t r\right)=\text { true } \vee e_{2}: p_{2}\left(e_{2} . a t t r\right)=\right.\right.\text { true } \\
& \left.\left.\vee \ldots \vee e_{n}: p_{n}\left(e_{n} . a t t r\right)=\text { true }\right\rangle\right\}
\end{aligned}
$$

A NOT (!) operator, which can occur within a $S E Q$ or $A N D$ operator, denotes the non-existence of an event. In $S E Q\left(E_{1}, \ldots\right.$, $\left.E_{i}, ! E_{j}, E_{k}, \ldots, E_{n}\right)$ a pattern match $\left\langle e_{1}, \ldots, e_{i}, e_{k}, \ldots, e_{n}\right\rangle$ is the output when no event instance $e_{j}$ exists in the stream such that $e_{i} . t s<e_{j} . t s<e_{k} . t s$. For $A N D\left(E_{1}, \ldots, E_{i}, ! E_{j}, E_{k}, \ldots, E_{n}\right)$ a pattern match $\left\langle e_{1}, \ldots, e_{i}, e_{k}, \ldots, e_{n}\right\rangle$ is the output if and only if no event instance $e_{j}$ exists in the stream in the interval [ $\left(t s_{c u r r}\right.$ Window), $\left.t s_{\text {curr }}\right]$.

Furthermore these operators can be nested within one another. For instance the query $S E Q\left(E_{11}, \ldots, E_{1 k}, A N D\left(E_{21}, \ldots, E_{2 n}\right)\right.$, $\left.E_{1 l}, \ldots, E_{1 m}\right)$ has an $A N D$ operator nested within a $S E Q$ operator. Semantically it refers to event patterns where $\left\langle e_{11}, \ldots, e_{1 k}\right.$, $\left.e_{1 l}, \ldots, e_{1 m}\right\rangle$ occur in order while events in the match $\left\langle e_{21}, e_{22}, \ldots\right.$, $\left.e_{1 n}\right\rangle$ occur in any order and $\forall e_{2 i}, e_{1 k} . t s<e_{2 i} . t s<e_{1 l} . t s$.

Predicate qualifications can be specified on the attributes of each event in the $S E Q, A N D, O R$ and NOT patterns. For instance, in Figure 1, $(C L$.pos $=\operatorname{toll} A)$ in $P 1$ represents a predicate specification on an event $C L$ that refers to a car passing a certain location on the highway.

The WITHIN clause defines the sliding window constraint common in continuous stream processing [27, 42]. The window parameter stipulates that the time difference between the first to the last event instances matched by a pattern query falls within the window constraint.

\subsection{Problem Statement}

Given a workload $W$ of pattern queries $\left\{P_{1}, P_{2}, \ldots, P_{k}\right\}$ specified in a stream $S$ and the statistics of the event stream, the goal of Sequence Pattern Sharing is to find a set of sub-patterns $\left\{S P_{1}\right.$, $\left.S P_{2}, \ldots, S P_{m}\right\}$ with $S P_{i}$ a sub-pattern of $P_{j}$ and $P_{j} \in W$ to share that minimizes computational costs for the entire workload $W$ and then to design a runtime infrastructure to concurrently execute the shared pattern plan achieving high processing performance.
Finding the optimal solution for this problem is prohibitively expensive as all combinations of possible sub-patterns form an exponentially large search space. Moreover, orchestrating a concurrent shared sequence pattern execution requires a meticulous yet lightweight transaction management mechanism to guarantee the result correctness.

\subsection{The SPASS Architecture}

Figure 2 shows the main components of the SPASS architecture. The SPASS architecture is composed of 2 modules - SPASS Optimizer and SPASS Runtime. The SPASS Optimizer takes as input a sequence pattern workload and outputs a sequence pattern sharing plan. The SPASS Runtime first instantiates this sequence pattern sharing plan on a concurrent execution infrastructure. Thereafter, it receives the event stream from external sources and produces pattern matches for the sequence pattern workload.

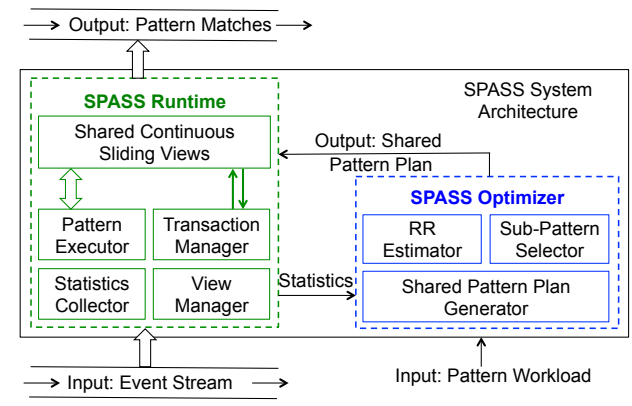

Figure 2: SPASS Architecture

\subsubsection{SPASS Optimizer}

The SPASS Optimizer adopts the principle of building an optimal sharing plan for the entire pattern workload [19] and then sharing similar components across these optimized queries when available [9]. To compute results for the workload of pattern queries with minimum CPU costs, the optimizer has to find a set of common sub-patterns that provides maximal sharing benefit.

RR Estimator. This module assigns a score referred to as $R e$ dundancy Ratio $(R R)$ to candidate sub-patterns based on CPU cost savings reaped when sharing them. This score is computed periodically using a cost model and event statistics collected by the Statistics Collector in the SPASS Runtime infrastructure.

Sub-Pattern Selector. This module uses $R R$ scores to identify the set of common sub-patterns to share across the pattern query workload. A polynomial-time algorithm produces a shared pattern plan covering the workload patterns with a bounded optimality.

Shared Pattern Plan Generator. The subset of sub-patterns chosen by the Sub-Pattern Selector is then transformed into a shared pattern plan by the Shared Pattern Plan Generator.

\subsubsection{SPASS Runtime}

The SPASS Runtime exploits stream transactions to support concurrent shared sequence pattern execution at runtime.

Pattern Executor. The Pattern Executor exploits the NFA-based pattern matching approach $[6,42]$ to compute pattern matches over event streams. It interacts with the View Manager to maintain and reuse sub-pattern matches in Shared Continuous Sliding Views.

Shared Continuous Sliding Views. The views are shared inmemory storage structures that store sub-pattern matches for reuse across multiple patterns. They are accessed by both the View Manager and Pattern Executor components. 
View Manager. The View Manager adopts the continuous sliding view technology [34] to compute and re-use pattern matches across multiple patterns. It is responsible for making decisions regarding storing, retrieving and updating sub-pattern matches in a shared in-memory storage structure.

Transaction Manager. Concurrently accessing and updating shared sub-pattern matches across pattern queries could result in missing or duplicate pattern matches if view reads and writes are not carefully controlled. The Transaction Manager is responsible for access control of these shared views.

Statistics Collector. The runtime statistics used by the $R R$ Estimator in the SPASS Optimizer are periodically collected by the Statistics Collector. Based on these periodic statistics, the SPASS Optimizer re-constructs a new sharing plan for the workload after these statistics are determined to have changed significantly [25].

\section{SPASS OPTIMIZER}

The goal of the SPASS Optimizer is to find a pattern sharing plan that minimizes the overall computational costs of the workload execution. To achieve this goal, we first establish a model to estimate the computational savings (Redundancy Ratio) achieved by sharing a specific sub-pattern. Second, we decide which subset of sub-patterns to share by reducing the well-known Minimum Substring Cover (MSC) [21] problem to our optimization problem. We exploit a polynomial-time algorithm for the MSC problem to efficiently find a near-optimal solution to our pattern sharing problem (Sec. 3.2). Lastly, we transform the selected sub-patterns into a hierarchical shared pattern plan covering all pattern queries in the workload (Sec. 3.3).

\subsection{Redundancy Ratio of Sub-patterns}

We now introduce the basis for the Redundancy Ratio $(R R)$ scoring model used to reflect the candidate sub-patterns' re-usability across the pattern workload. The lower the score, the more benefit we achieve by sharing this sub-pattern. The redundancy in computational costs due to a common sub-pattern depends on the number of sub-pattern matches that are common across a given set of queries. The redundancy ratio also depends on the event ordering within an input stream. We refer to this factor as the intra-query event correlation as formally defined in Def. 1. We also define the inter-query event correlation across patterns that impacts the number of sub-pattern matches that can be shared across multiple queries.

Intra-query Event Correlation is essential for estimating the number of sub-pattern matches that can be formed in a given time interval. Intuitively it represents the percentage of events of type $j\left(e_{j}\right)$ that follow an event of type $i\left(e_{i}\right)$. This ratio can help us estimate the number of matches produced by a sequence pattern.

DEFINITION 1 (INTRA-QUERY EVENT CORRELATION). For a time-ordered pattern $P=S E Q\left(E_{1}, \ldots E_{i}, \ldots E_{j}, \ldots, E_{n}\right)$, $C_{\text {Intra }}$ is defined as:

$$
C_{\text {Intra }}\left(E_{i}, E_{j}\right)=\frac{\left|\left\langle e_{i}, e_{j}\right\rangle\right|_{\text {window }}}{\left|e_{i}\right| *\left|e_{j}\right|_{\text {window }}}
$$

where $\left\langle e_{i}, e_{j}\right\rangle$ consists of $e_{i}$ and $e_{j}$ s.t $e_{i} . t s<e_{j} . t s$ and $\left|e_{i}\right|$ and $\left|e_{j}\right|$ denote the cardinality of event instances of type $E_{i}$ and $E_{j}$ respectively within one window.

Inter-query Event Correlation is used to estimate the sharing potential across multiple patterns. It is a ratio of the number of sub-pattern matches that are computed by two queries when they are shared to the total number of matches computed independently.
DEFINITION 2 (INTER-QUERY EVENT CORRELATION). Given an input stream and a common sub-pattern $S P$ of two patterns $P_{1}$ and $P_{2}$, the inter-query event correlation is defined as:

$$
C_{\text {Inter }}\left(P_{i}, P_{j}, S P\right)=\frac{\left|S P_{i, j}^{\text {share }}\right|_{\text {window }}}{\left|S P_{i, j}^{\text {noshare }}\right|_{\text {window }}}
$$

where $\left|S P_{i, j}^{\text {share }}\right|$ denotes the cardinality of the sub-pattern matches found in the input stream that qualify to form complete pattern matches in $P_{i}$ and $P_{j}$ in a shared fashion, and $|S P|_{i, j}^{\text {noshare }}$ is the sum of the cardinalities of $S P$ pattern matches produced by $P_{i}$ and $P_{j}$ separately.

Using the above defined notion of event correlation, our cost model estimates the costs for executing the sub-patterns in a shared versus an unshared manner. These cost functions are based on state-of-the-art physical implementations of operators as described in Appendix A.1. However, these functions could similarly be modeled for other operator implementations.

Time Period for Sampling. We propose to analyze the degree of sharing of sub-pattern matches over a certain time period, referred to as the sample time period. We then repeat this process on a periodic basis to accommodate event fluctuations. Within this time period, the optimizer constructs a stable sharing plan for the pattern workload. Choosing an appropriate sample time period has been well studied in the literature [16]. We leverage these techniques [16] to produce a stable execution plan designed to minimize CPU utilization. If the collected statistics significantly change, the SPASS Optimizer adapts to such change by migrating from the current shared pattern plan to the newly optimized one. For this we borrow plan adaptation techniques from the literature [31] to determine when plan migration becomes advantageous. Similarly, at the execution level we support caching for efficient plan migration [43] to avoid a full restart of the execution plan from scratch.

Analyzing the Cardinality of Sub-pattern Matches. Given a chosen sample time period, we compute the number of matches for a given sub-pattern within this time period. Figure 3 shows that the number of matches for a sub-pattern $S P=S E Q(A, B)$ produced by two parent patterns $P_{1}$ and $P_{2}$ may vary in a given time period. Consequently the number of pattern matches for $S P$ that can be shared across $P_{1}$ and $P_{2}$ also varies with time.

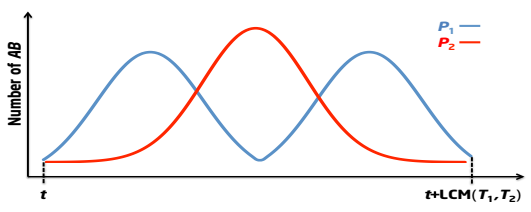

Figure 3: Non-Alignment of Cardinality of Pattern Matches

Observation 1. The cardinality of the sub-pattern matches varies over time. The crests and troughs of two such cardinalities plotted against time often may not align well. In fact, as depicted in Figure 3 , the crests and troughs of $P_{1}$ and $P_{2}$ never align in this particular scenario even if their average cardinalities over time happen to be similar. Hence, the inter-query correlation between $P_{1}$ and $P_{2}$ with respect to $A B$ would be quite low. Consequently, sharing this sub-pattern $A B$ between $P_{1}$ and $P_{2}$ may even cause more harm than good by incurring unnecessary concurrency control overhead.

Observation 2. Even when the cardinalities of the sub-pattern matches happen to be the same for the two pattern queries along the time-axis, this still does not guarantee re-use. Consider two patterns $P_{1}=S E Q(A, B, C, D)$ and $P_{2}=S E Q(A, B, X, Y)$ both 
with a constant window $=10 \mathrm{~s}$. The matches for $P_{1}$ are $\left\{\left\langle a_{2}, b_{3}\right.\right.$, $\left.\left.c_{5}, d_{10}\right\rangle,\left\langle a_{2}, b_{4}, c_{5}, d_{10}\right\rangle\right\}$ while those for $P_{2}$ are $\left\{\left\langle a_{5}, b_{7}, x_{9}\right.\right.$, $\left.\left.y_{15}\right\rangle,\left\langle a_{5}, b_{8}, x_{9}, y_{15}\right\rangle\right\}$. In this case, the sub-pattern SEQ(A,B) is not common across $P_{1}$ and $P_{2}$ at the event instance level. This confirms that the benefit of sharing $\operatorname{SEQ}(A, B)$ depends on the occurrences of the other sub-patterns in the parent pattern queries. We cannot solely rely on cardinality as decision criteria because the individual pattern matches may not actually overlap.

Redundancy Ratio Assignment. To accurately estimate the degree of the redundant computation of sub-pattern matches within each discrete time interval, we thus design a cost model $\mid C_{S}\left(S P_{i}\right.$, $\left.P_{m}, \ldots, P_{n}\right)\left.\right|_{j}$ that denotes the cost to compute matches of the shared sub-pattern $S P_{i}$ for all its parent patterns during the $j^{\text {th }}$ time interval. $\left|C\left(S P_{i}\right)\right|_{j}$ denotes the cost of producing all matches of $S P_{i}$ for each parent pattern separately. Hence, the computation reuse is essentially the difference between $\left|C\left(S P_{i}\right)\right|_{j}$ and $\left|C_{S}\left(S P_{i}, P_{m}, \ldots, P_{n}\right)\right|_{j}$. The Redundancy Ratio $R R$ of $S P_{i}$ at the $j^{\text {th }}$ time interval, denoted by $R R\left(S P_{i}\right)_{j}$, can be defined as:

$$
R R\left(S P_{i}\right)_{j}=\frac{\left|C_{S}\left(S P_{i}, P_{m}, \ldots, P_{n}\right)\right|_{j}}{\left|C\left(S P_{i}\right)\right|_{j}}
$$

$R R$ reflects the estimated cost benefit that can be achieved if subpattern $S P_{i}$ is shared among its parent pattern queries for a given interval $t_{j}$ in the event stream. The lower this $R R$ is, the higher the benefit of sharing this sub-pattern. We compute these costs for these non-overlapping discrete intervals. We then take the average of these scores over the entire sampled time period. This average score assigned to each sub-pattern $S P_{i}$, denoted by $R R\left(S P_{i}\right)$ is:

$$
R R\left(S P_{i}\right)=\frac{\sum_{j=0}^{t}\left[\frac{\left|C_{S}\left(S P_{i}, P_{m}, \ldots, P_{n}\right)\right|_{j}}{\left|C\left(S P_{i}\right)\right|_{j}}\right]}{t}
$$

where $t$ denotes the number of discrete intervals in the sampled time period. The number of discrete intervals is referred to as the level of granularity of discretization. Fine-grained analysis of sub-patterns highlights the actual re-usability of sub-pattern results

\begin{tabular}{|c|c|c|c|c|c|c|c|c|}
\hline & & $t_{1}$ & $t_{2}$ & $t_{3}$ & $t_{4}$ & $t_{5}$ & $T$ & \\
\hline \multirow{3}{*}{$A B$} & $P_{1}$ & 400 & 500 & 300 & 100 & 50 & 1350 & \multirow{2}{*}{$\begin{array}{c}\text { No } \\
\text { Sharing }\end{array}$} \\
\hline & $P_{2}$ & 100 & 200 & 300 & 500 & 300 & 1400 & \\
\hline & $P_{1} \cup P_{2}$ & 400 & 500 & 300 & 500 & 300 & 2000 & Sharing \\
\hline \multirow{3}{*}{$X Y$} & $P_{1}$ & 400 & 500 & 300 & 400 & 50 & 1650 & \multirow{2}{*}{$\begin{array}{c}\text { No } \\
\text { Sharing }\end{array}$} \\
\hline & $P_{2}$ & 100 & 100 & 100 & 500 & 300 & 1100 & \\
\hline & $P_{1} \cup P_{2}$ & 400 & 500 & 300 & 500 & 300 & 2000 & Sharing \\
\hline
\end{tabular}
across the parent queries.

Figure 4: Example of Discretized Granularity

EXAMPLE 1. Consider the following two sub-patterns $A B$ and $X Y$. Assuming that the sampled period for both $A B$ and $X Y$ is $T$, and $T$ is discretized into 5 time intervals (i.e., $t_{1}, t_{2}, t_{3}, t_{4}$, and $t_{5}$ ). The number of matches computed with or without sharing in these intervals are shown in Figure 4.

Now if we were to treat the entire period $T$ as one time interval, then $R R(A B)=2000 /(1350+1400)=R R(X Y)=2000 /(1650+$ $1100)=0.727$. In contrast, if the RR of both sub-patterns were to be calculated with finer granularity, the $R R$ of $A B$ and $X Y$ would be 0.741 and 0.759 , respectively. Clearly sharing the sub-query $A B$ would gain more computational savings compared to XY. Hence, the cost derived with a fine granularity of discrete time intervals more accurately reflects the degree of sharing.
Sharing Sub-patterns Across Multiple Window Sizes. Multiple window sizes for event patterns are easily accommodated in the Redundancy Ratio computation. When queries have different window sizes, we use the Lowest Common Multiple (LCM) of the window sizes as the smallest unit of time during which each query produces pattern matches for an integral number of window slides.

\subsection{Sharing Sub-Patterns to Minimize Redundancy in Computation}

Having the $R R$ score assigned to each sub-pattern in the above step, the SPASS Optimizer needs to find the most suitable subset of sub-patterns to share. Given a set of pattern queries $W$, the sub-pattern sharing optimization problem is to find a subset of sub-patterns such that all pattern queries in the workload $W$ can be answered, and the total redundancy ratio of this subset is minimal compared to all possible alternate solutions. We prove that this problem is equivalent to the well-known Minimum Substring Cover problem [21]. The SPASS Optimizer can thus employ a polynomial-time approximate solution to the Minimum Substring Cover problem to produce the set of sub-patterns to share. We now describe how the sequence sub-pattern sharing problem can be mapped to the Minimum Substring Cover Problem. In later subsections we extend this to consider other operators.

Minimum Substring Cover (MSC). For a given set of strings $S$, let $\Theta(S)$ denote the set of all substrings of strings in $S$. We define a cover of $S$ to be a subset $C \in \Theta(S)$ such that any string $s \in S$ can be written as a concatenation of strings in $C$. The MSC problem is formally defined as follows.

Definition 3 (Minimum SUbSTRING COVER). Instance: A set of strings $S$, a weight function $F: \Theta(S) \rightarrow \mathbb{Q}_{0,1}$ where $\mathbb{Q}_{0,1}$ is the set of positive proper fractions. Solution: A cover $C$ of $S$. That is, a set of strings $C \subseteq \Theta(S)$, where for each $s \in S$ a set $c_{1}, \ldots, c_{p} \subset C$ exists, with $s=c_{1} \bullet \ldots \bullet c_{p}$ where $\bullet$ represents concatenation. Measure: The total weight of the cover, i.e., $F(C)=\Sigma_{c \in C} F(c)$ is minimal among all possible covers.

Problem Mapping. We now show that having assigned weights to the sub-patterns which have the same properties as those of the weights of the substrings, the sub-pattern sharing problem is equivalent to the Minimum Substring Cover problem.

Lemma 1 (REDUCTION). If the Redundancy Ratio $(R R)$ of the sub-patterns is in $\mathbb{Q}_{0,1}$, then every instance of the sub-pattern sharing problem can be reduced to a valid instance of the Minimum Substring Cover (MSC) problem (Def. 3).

The proof can be found in Appendix A.4.

Approximate Algorithm to MSC. The well-known Local-Ratio (LR) algorithm [21] has been shown to find a cover of a set of strings $C$ in polynomial time. Furthermore, it guarantees an approximation factor of $m$ where $m$ is the maximum length of the strings in the set of strings. We now leverage this algorithm to find the approximate set of sharing sub-patterns. The algorithm takes as input the pattern workload $W$ and our $R R$ scores of its sub-patterns. We output a list of pattern queries and their associated sub-patterns as a query map $\left(q M a p\left\langle P_{i}, \operatorname{list}\left\langle S P_{i}\right\rangle\right\rangle\right)$. This data-structure subsequently facilitates easy construction of the $h i$ erarchical execution plan. We denote the set of all sub-patterns as $\Theta(W)$ and each individual sub-pattern in $\Theta(W)$ as $S P_{i}$. The solution $q M a p$ is first set as empty (Line 1). The algorithm selects a pattern $P_{i}$ not covered by $q M a p$ (Line 3 ), and examines all sub-patterns in a temporary structure $q M a p^{c}$ that are not already in $q M a p$ (Lines 4$)$. It then subtracts the minimum score $\epsilon=$ 
$\min \{R R\}$ from the cumulative $R R$ (Lines 7), and recurs over the new weight function (Line 8 ).

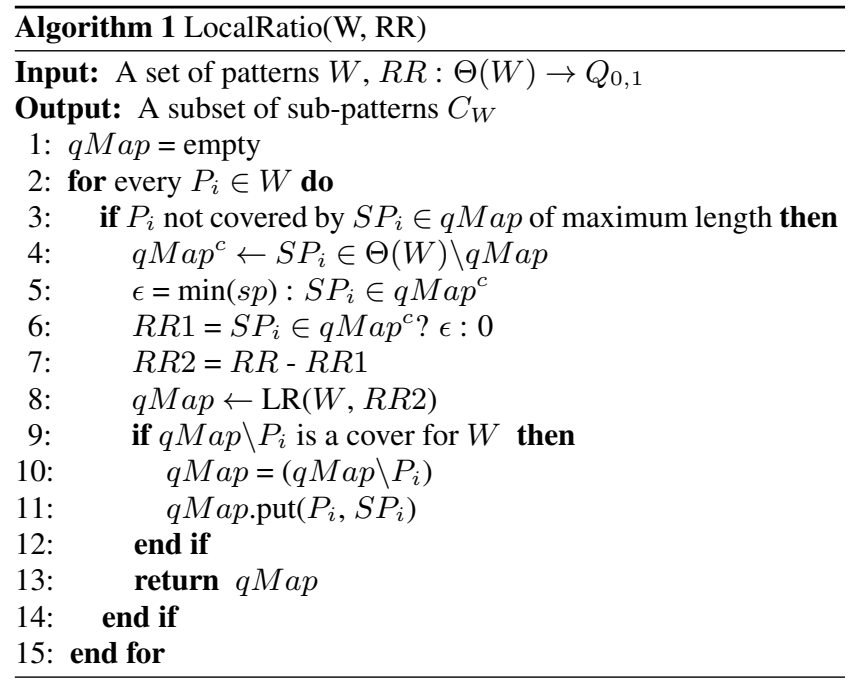

\subsubsection{Sharing Across Other Operators}

The SPASS system supports a variety of event pattern operators including $O R, A N D$ and NOT with its optimization strategy described above with minor to no alteration. Both negation in $S E Q$ or in $A N D$ patterns like $! E_{i}$ can be considered as a new symbol. Thus before using the solution to the MSC problem, each negated event type is mapped to a particular letter. The OR operator is simply a filter. Therefore it can be easily piggy-backed as part of the pattern detection process. We now describe how pattern matches can be shared across $S E Q$ and $A N D$ operators.

While the previous derivation has been shown for sharing across multiple $S E Q$ operators, the same can be extended to accommodate the $A N D$ operators. In essence an $A N D$ operator is a permutation of a set of events. Thus a $S E Q$ and an $A N D$ operator with the same set of events would have some matches in common. For instance $A N D(A, B)$ will produce matches for events $A$ and $B$ arriving in any order within the window, while $S E Q(A, B)$ will produce matches for events $A$ and $B$ arriving in order within the same window. Thus a subset of matches for $A N D$ operator will also qualify as matches for the $S E Q$ operator. We can therefore share pattern matches between these different operators.

Our assumption here is that the most common type of CEP operator is the $S E Q$ operator along with some $A N D$ operators. Hence given a workload composed of many $S E Q$ and some $A N D$ operators, we slightly modify the above problem mapping to share pattern matches across both $S E Q$ and $A N D$. One important observations is that only those $A N D$ pattern matches can be shared with a $S E Q$ operator that have events arriving in the same order specified in the $S E Q$ operator. Thus as a first step to the MSC problem we rewrite the $A N D$ operator such that the events align with the $S E Q$ operators. Given a set of $m S E Q$ operators and $k$ $A N D$ operators, we rewrite each $A N D$ operator in a maximum of $m$ distinct ways such that each aligns with the operands in each $S E Q$ operator. Using this rearrangement, it is now possible to see the overlap of event sub-patterns across $S E Q$ and $A N D$ operators using the solution to the MSC problem. Thus the search space is only increased by a polynomial of $m k$ rather than an exponential increase incurred by enumerating all permutations of $A N D$.

The weights of sharing these sub-patterns across different operators correspond to the ratio of how many pattern matches are shared to the total number of pattern matches. Thus using the previous example of $S E Q(A, B)$ and $A N D(A, B)$, if most pattern matches for $A N D$ are such that $B$ arrives after $A$, then the Redundancy Ratio correctly reflects that with a lower score.

During execution, when $S E Q$ and $A N D$ operators are shared, the implementation of the $A N D$ operator is deployed and the set of pattern matches arriving in the same order as the $S E Q$ are pushed to the $S E Q$ query. If multiple orderings of the same operands of the $A N D$ operator are chosen, they are identified in the step of building the shared pattern plan and are shared by multiple $S E Q$ operators.

EXAMPLE 2. Given $S E Q(X, B, A, Y, C, E), S E Q(P, E, A, B$, $Q), S E Q(D, E, B, A, C)$ and $A N D(A, B, C, D, E)$. Here the $A N D$ query is rewritten as: $A N D(D, B, A, C, E), A N D(C, D, E, A, B)$ and $A N D(D, E, B, A, C)$. Thus there are now 6 patterns instead of 4 patterns as input to the MSC problem.

\subsection{Shared Pattern Plan}

The solution to the minimum substring cover problem returns a map of pattern queries with their corresponding set of sub-patterns that cover this pattern query $\left(\operatorname{map}\left\langle P_{i}, \operatorname{list}\left\langle S P_{i}\right\rangle\right\rangle\right)$. We now generate a shared pattern plan that compactly represents the multiple pattern workload. Algorithm 2 transforms the set of sub-patterns into a shared pattern plan. Such transformation-based approach is commonly used to generate shared query plans for traditional multi-query workloads $[17,24]$.

The shared pattern plan is represented as a graph $q$ Graph. Each node in the graph represents a pattern or sub-pattern. Algorithm 2 iterates over the patterns in $q \operatorname{Map}\left\langle P_{i}, \operatorname{list}\left\langle S P_{i}\right\rangle\right\rangle$. For each pattern $P_{i}$, if a corresponding node is not found in $q G r a p h$, a root node is created. Its corresponding sub-patterns are scanned and the following action is taken: if a sub-pattern $S P_{j}$ corresponds to an existing node in $q G r a p h$, no action is required. If a sub-pattern is seen for the first time, it is added to the $q$ Graph as a child node of $P_{i}$. We illustrate Algorithm 2 using the following example.

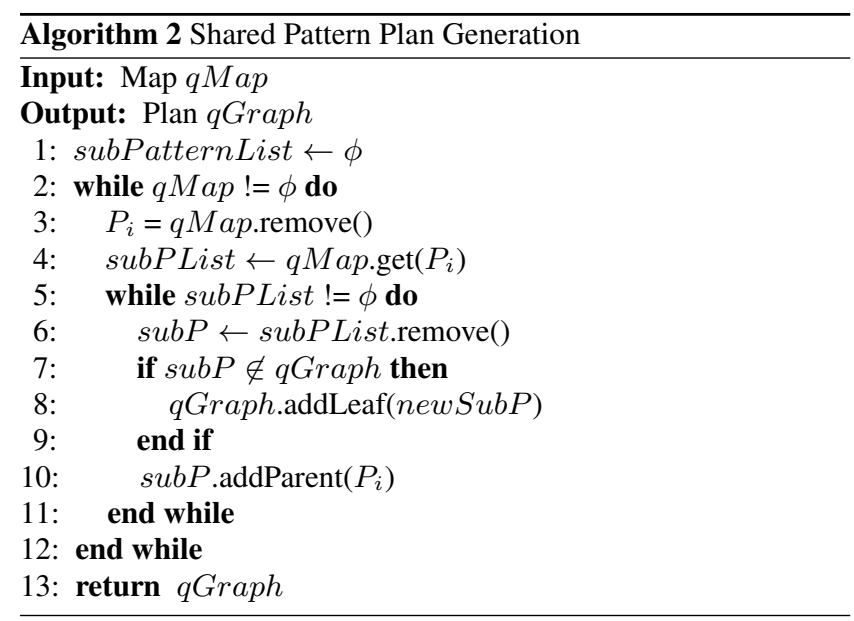

EXAMPLE 3. The following three pattern queries (i.e., $P_{1}, P_{2}$, and $P_{3}$ on the left) and the selected sub-patterns for sharing (on the right) are the inputs to our SPASS Optimizer.

$P_{1}:(\operatorname{SEQ}(A, B, C)) \rightarrow \operatorname{SEQ}(A, B), S E Q(B, C)$

$P_{2}:(S E Q(A, B, C, D)) \rightarrow S E Q(A, B, C), S E Q(D)$

$P_{3}:(S E Q(A, B, X)) \rightarrow \operatorname{SEQ}(A, B), S E Q(X)$

Given the above selected sub-patterns to share, the algorithm loops through the patterns from $P_{1}$ to $P_{3}$. It scans the shortest sub- 
patterns of $P_{1}$ which are $\operatorname{SEQ}(A, B)$ and $\operatorname{SEQ}(B, C)$. Therefore they are made leaves in the pattern DAG. SEQ $(A, B, C)$ is made the parent of these leaves. For $P_{2}$, the shortest sub-pattern $S E Q(D)$ is made a leaf. The next shortest pattern $S E Q(A, B, C)$ is then found to be already in the pattern DAG. Hence $S E Q(A, B$, $C, D)$ is made the parent of $S E Q(A, B, C)$ and $S E Q(D)$. For $P_{3}$, $S E Q(X)$ is added as a leaf node and $S E Q(A, B)$ already exists in the DAG. The final query DAG formed is depicted in Figure 5.

Figure 5: Shared Pattern Plan

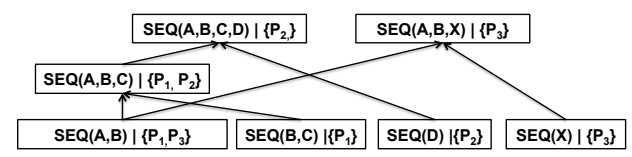

type. All patterns composed of this event type access this shared buffer during the pattern matching process. On the other hand the shared continuous sliding views (S-CSV) cache intermediate results for sub-patterns. Each shared sub-pattern $S P_{i}$ in the shared pattern plan has an associated S-CSV structure denoted by view $w_{S}$ that maintains matches for the sub-pattern $S P_{i}$.

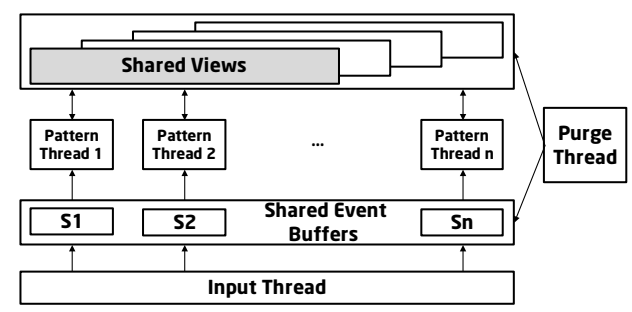

Figure 6: Shared Pattern Executor

\section{SPASS RUNTIME}

Given a shared pattern plan produced by the SPASS Optimizer, we now introduce the SPASS Runtime - our multi-threaded execution engine for concurrent shared pattern processing on event streams. We first describe the execution strategy for processing this plan assuming that only one pattern is processed upon the arrival of an event instance. Thereafter, we relax this restriction to handle (1) multiple events and (2) multiple patterns triggered simultaneously.

The key challenge of maintaining result matches for sub-patterns is tackled by plugging in continuous sliding views technology [34], which stores intermediate results of sub-patterns and incrementally updates and reuses them for answering a single sequence pattern query over sliding windows. We adopt and then extend this view technology from supporting a single pattern query to shared continuous sliding views to handle our multiple pattern scenario. We assume an identical window constraint of size window which later is relaxed.

\subsection{Shared Pattern Execution}

SPASS Execution Model. The SPASS Runtime receives event tuples from their respective input event threads. The events are assumed to arrive in order. Each event type is associated with a shared event buffer. When an event arrives, it is inserted into its associated shared event buffers. Each pattern designates a triggering event that initiates the execution of this pattern. For the $S E Q$ pattern $S E Q(A, B, C)$, the triggering event is $C$, which implies that the execution of $S E Q(A, B, C)$ begins only when an event of type ' $C$ ' arrives. When a sequence pattern execution is triggered by the arrival of its triggering event, all its sub-patterns are executed. Our execution adopts a Non-deterministic Finite Automata (NFA) approach to represent the structure of an event sequence. Namely, sequence patterns are computed using the NFA-based execution strategy by mapping successive event types to successive NFA states [6, 42]. Each sub-pattern execution is an independent thread that reads events from the shared event buffers. These partial sub-pattern matches are then stored in shared storage structures described next. These matches are not all computed from scratch. Instead sub-pattern matches that have once been computed are then stored in sequence views and subsequently reused by accessing the materialized views associated with that sub-pattern.

Shared Storage of Events and Intermediate Pattern Matches. Two types of shared storage units are used in the SPASS Runtime infrastructure (Figure 6). As is common in CEP systems [42], we utilize shared event buffers to store event instances for each event
Actions on Event Arrival. As events arrive into the SPASS Runtime, one of several actions is triggered. (1) Append. The arriving event is inserted into the respective event buffer via an Append operation on the shared event buffer. (2) Read. Pattern execution of a pattern $P_{i}$ is triggered if the event is a triggering event. During pattern execution, events are read from shared event buffers and views as needed. These are modeled as Read operations. Subpattern matches are inserted into the corresponding shared views during pattern execution. This is modeled as an Append operation on the continuous sliding view structure. (3) Purge. Events from the buffers or sub-pattern matches in the shared views eventually expire out of the current window. They then should be purged. Assuming the new event $e_{i}$ arrives at time $i$ all events with timestamps less than $(i-$ window $)$ are deleted*. This is modeled as a Purge operation. In summary, three types of operations, Read, Append, and Purge, are performed on the two types of shared memory structures, namely shared event buffers and shared continuous sliding views.

Pattern Execution via Shared Continuous Sliding Views. If a pattern $P_{i}$ execution is triggered by the arrival of an event $e_{i}$, all its sub-patterns $S P_{i}$ are executed one by one until completion. $S E Q$ sub-patterns are computed using the NFA-based execution strategy $[42,6]$ while $A N D$ sub-patterns are executed using a traditional join operator. The sub-pattern matches are however not all computed from scratch. Instead sub-pattern matches that had previously been computed are stored in a view associated with that subpattern. These sub-pattern matches stored in the views can then be shared across multiple patterns. Thus matches for sub-pattern $S P_{i}$ produced and stored by one pattern $P_{j}$ could be reused by another pattern $P_{k}$.

In order to facilitate collaborative re-use of shared continuous sliding views, we augment these view structures with timestampbased indicators called the View Validity Interval (VVI). These VVI indicate the interval of time for which the buffer contains the complete set of event matches. Further, when a pattern accesses these view structures, it looks for pattern matches within a certain time interval called the View Lookup Interval (VLI). [34]. Based on whether this interval is currently valid for the shared view, pattern matches are read from the view $\left(\operatorname{Read}_{V i e w}\right)$ or new matches are written to the view $\left(A p p e n d_{V i e w}\right)$. When new matches are appended to a view, the $V V I$ is updated to reflect the latest state of the view. Example 4 demonstrates the above principles.

\footnotetext{
*We assume that events arrive in increasing order of timestamp.
} 
EXAMPLE 4. Consider the patterns $P_{1}$ and $P_{2}$ depicted in Figure 7. $P_{1}$ and $P_{2}$ both share the sub-pattern $S P_{c}$ using its associated shared view views $S_{S c}$. For pattern $P_{1}, S P_{1}$ is executed followed by $S P_{c}$ while for pattern $P_{2}, S P_{2}$ is executed followed by $S P_{c}$. Each pattern has a designated triggering event type ( $D$ for $P_{1}$ and $Y$ for $\left.P_{2}\right)$. When event instance $y_{13}$ arrives, $P_{2}$ is triggered and a match $\left\{\left\langle x_{5} y_{13}\right\rangle\right\}$ is produced for sub-pattern $S P_{2}$ of $P_{2}$. Then sub-pattern $S P_{c}$ is executed for the constrained View Lookup Interval (VLI) in this case is [13-10, 5] The view is thus updated to contain matches valid for the interval [3,5]. As the triggering event $d_{17}$ for $P_{2}$ arrives, the match $\left\{\left\langle c_{16} d_{17}\right\rangle\right\}$ is produced for subpattern $S P_{1} . S P_{c}$ is now updated for the interval [7, 16]. Four new sub-pattern matches are produced and stored in the view ${ }_{S C}$ for possible reuse. The view interval is now set to [7,16] to mark its completeness up to 16 . Upon the arrival of event instance $d_{18}$, the view content is reused. Furthermore, when $y_{20}$ arrives, $P_{2}$ triggers the update of $S P_{c}$ for the interval [10, 18]. This process illustrates how we have step by step supported synchronous reuse and update of the views.

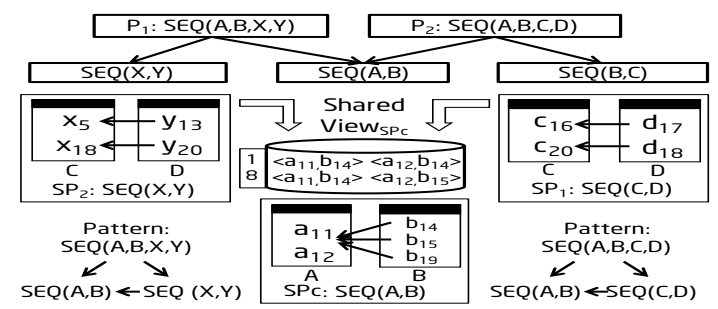

Figure 7: Shared $S E Q$ Execution using Continuous Views

For simplicity we have described the execution when all patterns share the same window and no predicates exist. However multiple windows can be easily supported by keeping pattern matches for the largest window. Each pattern then filters out the matches relevant to its specific window.

The reuse, append, and purge of the shared continuous sliding view may require an additional step to ensure the result correctness based on the largest window among all windows associated to a view. Similarly, correlated predicates in the patterns can also be supported by an additional filtering phase. We adopt popular approaches for handling predicate sharing from the literature [20, 27]. For example, if a sub-pattern $S P$ is shared across two patterns yet the two patterns specify different selection predicates $C_{1}$ and $C_{2}$ on this sub-pattern respectively, then the shared sub-pattern is rewritten as $\left(C_{1} \vee C_{2}\right)$ to satisfy either selection predicate using well-known query rewriting techniques [27] and thus to be sharable. Predicate migration techniques such as push-down and pull-up [20] can be exploited in our SPASS Runtime as well. Further details are described in Appendix B.2.

\subsection{Concurrent Shared Execution}

We now relax the assumption that only one event is processed at a time until its completion. Instead we now consider the concurrent execution of two or more input events. As a consequence multiple pattern queries may be active at the same time. These concurrent execution threads could therefore attempt to access or update a shared continuous sliding view at the same time. This may lead to duplicate or missing results in the view.

EXAMPLE 5. Figure 8 shows a time instance when the shared view contains all results valid during the time interval [5,10]. At

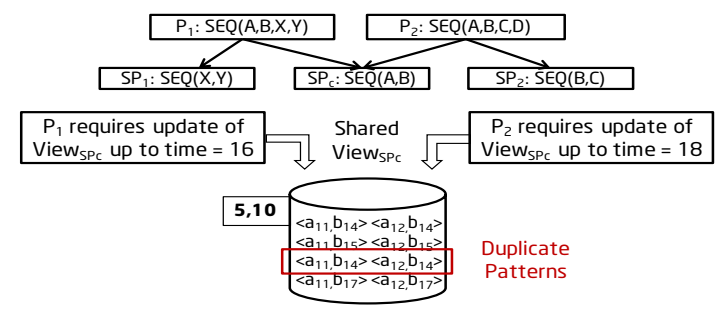

Figure 8: Duplicate Pattern Matches due to Concurrency

this instance, pattern $P_{1}$ requires to append the shared view up to time 16. At the same time $P_{2}$ is triggered and tries to also update the view up to timestamp 18. Without careful scheduling, $P_{2}$ might start updating while $P_{1}$ is still updating the view. This results in duplicate matches being produced namely $\left\{\left\langle a_{11} b_{14}\right\rangle,\left\langle a_{12} b_{14}\right\rangle\right\}$ as well as resources being wasted due to repeated computations.

The above example demonstrates how duplicate pattern matches may be produced during concurrent shared pattern execution. Thus our SPASS Runtime needs to deploy a concurrency control mechanism that avoids these erroneous results. As explained below, we use a transaction-based approach to guarantee correctness.

In the SPASS system, each event has a single point of entry. On arrival an event can trigger one or more of the following types of actions, namely, $R_{\text {View }}, A_{\text {View }_{t}}, P_{\text {Viewt }_{t}}, R_{\text {Buffer }}, A_{\text {Buffert }}$, and $P_{\text {Buffer }}(R-$ Read, $A-$ Append, $P-$ Purge $)$. Given the indicators attached to shared views, namely the View Validity Intervals, we determine upon the entry of an event which data structures the event will need to read from and which it will need to update.

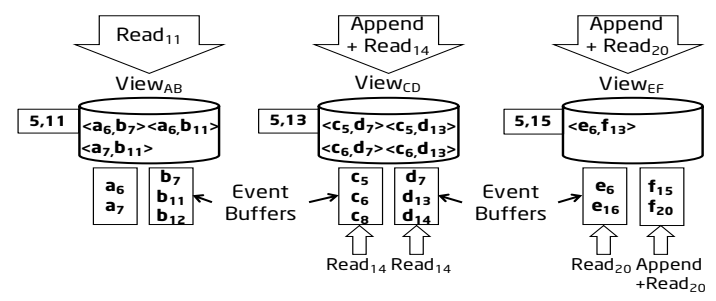

Figure 9: Actions on Event Buffers and Views

EXAMPLE 6. Figure 9 depicts a pattern $S E Q(A, B, C, D, E, F)$ that is partitioned into three parts namely $S E Q(A, B), S E Q(C, D)$ and $S E Q(E, F)$. When the event $f_{20}$ arrives, the View Validity Intervals (VVIs) associated with the views View $w_{E}, V i e w_{C D}$, and $V_{\text {ie }} w_{B}$ are [5,15], [5,13], and [5,11], respectively. Judging from the shared event buffers it can be inferred that the View ${ }_{E}$ must be updated up to the time 20. In fact, the event buffer F must be updated as well as read from. The event buffer $E$ must only be read from. Consequently, the event buffers of $C$ and $D$ must be read to update the View $w_{C D}$ accordingly. The View $w_{C D}$ will be updated to the time 14 as $d_{14}$ is the last event in $D$ that can join with the event instance $e_{16}$ in $E$. For the View $w_{A B}$, we can infer that it only needs to be read from as the last $B$ event that can join with a $C$ event to form valid matches is $b_{11}$. The View $w_{A B}$ already contains matches up to this timestamp 11. Hence, the event buffers $A$ and $B$ do not need to be read from. Thus the actions triggered upon the arrival of event $f_{20}$ are $A_{F_{20}}, R_{F_{20}}, R_{E_{20}}, A_{E F_{20}}, R_{E F_{20}}, R_{C_{14}}, R_{D_{14}}$, $A_{C D_{14}}, R_{C D_{14}}, R_{A B_{11}}$. Finally the last action is to purge events 
beyond the current window given by currentTime - window $=$ $20-15=5$, namely events with timestamp $<5$.

Notion of Correctness. As seen from the above example multiple actions are triggered upon the arrival of an event. Several of these actions can take place out of order, while for others a strict order must be enforced. These semantics include:

1. $A_{V i e w}$ must be preceded by $R_{B u f f e r s}$. In order to append results to a given view, its associated event buffers must first be read. 2. If there are two append actions on different views $A_{V i e w 1}$ and $A_{V i e w 2}$, any order of execution would be correct.

3. $P_{\text {View }_{t}}$ or $P_{\text {Buffer }_{t}}$ can be executed only when all operations before timestamp $t$ have been completed.

The action $A_{V i e w}$ requires new pattern matches to be constructed and written to the view. Thus it needs to first read events from multiple event buffers $\left(R_{B u f f e r(s)}\right)$. Hence $\left\{R_{B u f f e r(s)}+A_{V i e w}\right\}$ must occur in order. On the other hand the actions $A_{B u f f e r}, R_{V i e w}$, $P_{B u f f e r}, P_{V i e w}$ are independent of each other and thus can be executed in any order.

DEFINITION 4. A SPASS Transaction is defined as the minimal set of SPASS actions that must be enforced to occur together as an atomic series of operations when an event arrives.

Analysis of the SPASS Runtime reveals that there are the following set of atomic actions namely: $\left\{R_{V i e w}\right\},\left\{A_{V i e w}+R_{B u f f e r(s)}\right\}$, $\left\{A_{B u f f e r}\right\},\left\{P_{V i e w / B u f f e r}\right\}$. Multiple transactions can be triggered upon the arrival of an event. They would each belong to one of the above four types of transactions. The above definition considers the notion that when a view is updated, it must be able to access the event buffers which form the raw events for the pattern matches. On the other hand, if an event triggers appends for two views, the two appends can take place completely independent of each other, thus allowing maximum parallelism. Further, the $R_{V i e w}$ transaction is separated from $A_{V i e w}$ to allow higher degree of parallelism as multiple $R_{V i e w}$ transactions can occur simultaneously while only one $A_{V i e w}$ is allowed on a view.

Lock Insertion and Compatibility: Locks are inserted upon the arrival of an event into the SPASS system. As shown in Example 6, the actions to be triggered in the SPASS system are determined as soon as an event arrives into the system. These actions are grouped into valid classes of transactions. Thus the locks required by these transactions can also be determined at this stage. These locks are then inserted into lock buffers associated with each shared storage i.e., event buffers and shared views. Thus a purge request $P_{*_{t}}$ will be inserted only when requests for locks triggered by events arriving before $t$ have all been inserted into lock buffers. This purge lock will not be granted until all other lock requests with an earlier timestamp have been processed.

Once locks have been inserted into respective lock buffers the atomic set of actions are ready to be triggered. Henceforth we adopt the well-known scheduling scheme of Strict 2-Phase Locking (S2PL) [38]. There is a pool of atomic actions that exist on a given shared buffer at any given time. Based on the lock compatibility protocol described below, some will be allowed to run. Figure 10 shows the compatibility of Read, Append and Purge locks. The read and append locks on shared buffers are completely independent of each other due to the sequential lock assignment. For shared views, append operations are not allowed to be executed concurrently. This avoids the creation of duplicate results. Lastly, the purge lock on shared buffers and views are most restrictive. In order to grant a purge request, the transaction manager must first make sure that the lock buffer for that resource does not contain any read requests on that resource that may try to read events or pattern matches purged by this purge transaction. This prevents any missing pattern matches to arise.

\begin{tabular}{|l|c|c|c|c|}
\hline \multirow{2}{*}{$\begin{array}{l}\text { Acquired } \\
\text { Requested }\end{array}$} & \multicolumn{2}{|c|}{ Shared Buffer } & \multicolumn{2}{c|}{ Shared Views } \\
\cline { 2 - 5 } & Read & Append & Read & Append \\
\hline Read & $\boldsymbol{V}$ & $\boldsymbol{V}$ & $\boldsymbol{V}$ & $\boldsymbol{V}$ \\
\hline Append & $\boldsymbol{V}$ & $\boldsymbol{V}$ & $\boldsymbol{V}$ & $\mathbf{X}$ \\
\hline Requested \\
\hline Purge & $\mathbf{X}$ & $\boldsymbol{V}$ & $\mathbf{X}$ & $\boldsymbol{V}$ \\
\hline
\end{tabular}

Figure 10: Lock Compatibility in Transactions

\section{PERFORMANCE EVALUATION}

\subsection{Experimental Setup}

We have implemented our SPASS framework on top of the Chaos stream management system [40] using Java. Experiments were conducted on a PC with Intel Pentium IV 2.8 GHz CPU and 4GB RAM running Windows 7 system.

Methodology. We evaluate the efficiency and plan quality produced by our SPASS Optimizer. We compare our shared multiple sequence pattern execution strategy to the state-of-the-art approaches, including single sequence pattern query [42] evaluation without sharing sub-patterns, and shared sequence pattern query evaluation via prefix and suffix sharing [14]. Next we evaluate the efficiency and scalability of our SPASS Runtime by varying various parameters of the workload patterns. We show the performance benefits of the SPASS Runtime on a multi-core architecture by running it in single and multi-threaded mode.

Data sets and queries. We evaluate our techniques using two data sets, (1) real life stock trades data [1], which contains stock ticker and timestamp information, and (2) traffic data set using the data generator of the linear road [2]. In stock dataset, the portion of the trace holds 120,000 event instances. Each event has a stock ticker name and a timestamp information. To evaluate SPASS we create event patterns over these data sets, including $S E Q, A N D$, and ! operators. We show experiments with the traffic dataset in the Appendix C.

Evaluation metrics. We compare the average execution time for computing multiple sequence pattern queries per window slide. After every window slide, the execution time is recorded for each pattern and averaged over the entire pattern workload run time. The average execution times for all patterns are then added to produce the average execution time per window for the entire workload.

\subsection{Evaluating SPASS Optimizer}

Here, we study the effectiveness and accuracy of our SPASS Optimizer for shared multiple plan generation. Specifically, we compare the compile-time optimization performance and the quality of the resulting plans for two alternative techniques, namely the approximate LR algorithm $(L R)$ and exhaustive search $(E S)$. The compile-time optimization performance is measured by the CPU time taken by either algorithm. We vary the key parameters that affect the performance of our SPASS Optimizer including the length and the number of the patterns. As baseline for the best quality shared pattern plan, we employ $E S$ for finding the optimal plan for the given pattern workload. We generate pattern queries over the stock stream [1] data set to evaluate the SPASS Optimizer. Two types of workloads, only $S E Q$ patterns as well as mixed patterns composed of $S E Q$ and $A N D$, are used to evaluate our optimizer. 


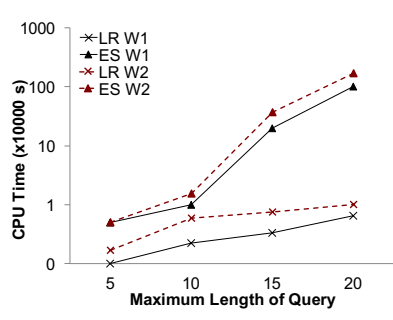

(a) Varying Pattern Length $(l)$

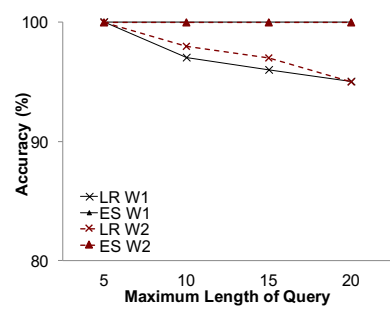

(b) Varying Pattern Length $(l)$

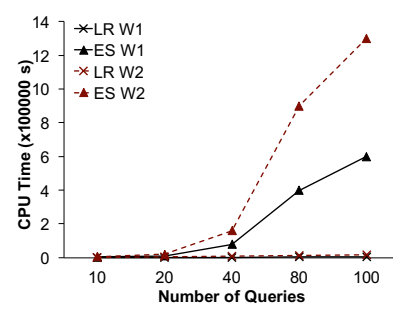

(c) Varying Pattern Number $(k)$

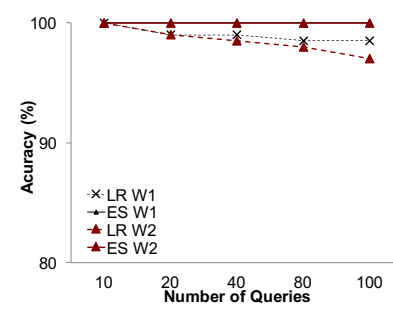

(d) Varying Pattern Number $(k)$

Figure 11: Efficiency and Quality of SPASS Optimizer

\subsubsection{Effect of Varying the Pattern Length $l$}

Efficiency of SPASS Optimizer. We first evaluate the efficiency of the SPASS Optimizer with respect to plan generation time. We consider a workload of 10 patterns in which the length of the sequence pattern in each pattern is varied from $l=5$ to 20 . The continuous lines correspond to the workload with only $S E Q$ queries while the dotted lines correspond to the mixed workload with $S E Q$ and $A N D$ patterns. Our optimizer using the $L R$ algorithm, as shown in Figure 11(a), is significantly more efficient than the alternative $E S$ approach for both workloads. The total number of possible sub-patterns is proportional to the square of the length of the sequence pattern [21]. This drastically affects $E S$ because this approach has to check all possible combinations in the search space. As depicted in Figure 11(a), the optimization time of ES increases exponentially (around 1,000,000 times) as the pattern length is increased from 10 to 20 . In contrast, our proposed $L R$ algorithm increases polynomially with the pattern length by a factor of around 6 times. The workload with mixed $A N D$ and $S E Q$ patterns also maintains approximately the same ratio for $E S$ and $L R$ approaches.

Plan quality of SPASS Optimizer. Figure 11(b) shows the quality of the set of sub-patterns produced by our $L R$ algorithm. The quality of the shared pattern plan is obtained by the total $R R$ of the sub-patterns selected by the optimizer. The optimal solution should give the minimum total $R R$. As expected, the quality of the subpattern set from the $L R$ algorithm slightly degrades as the length of the patterns increases. Compared to $E S$, the quality suffers from the degradation by $5 \%$ as the length of the sequence pattern increases from 10 to 20 . In this case both workloads approximately has the same reduction in accuracy.

\subsubsection{Effect of Varying the Number of Patterns $k$}

Efficiency of SPASS Optimizer. We now examine the efficiency of the optimizer as the number of patterns varies from 10 to 100 while keeping the length of each pattern constant at 15 events. Our optimizer, as shown in Figure 11(c), is significantly more efficient than the $E S$ approach. Again, the total number of possible subpatterns is proportional to the number of patterns in the worst case. As depicted in Figure 11(c), ES is prohibitively expensive and thus is not practical. The optimization time of $E S$ increases exponentially (around 400,000 times) as the number of patterns increases from 40 to 100 . In contrast, our proposed $L R$ optimizer increases linearly by wisely choosing the sub-patterns with lower redundancy $R R$ (Section 3.1). Again, the mixed workload (depicted by the dotted lines) takes overall higher optimization time due to the extra search complexity incurred by $A N D$ patterns.

Plan quality of SPASS Optimizer. Figure 11(d) compares the quality of the set of sub-patterns produced by our $L R$ algorithm with the optimal solution produced by $E S$ for different numbers of patterns. As depicted in Figure 11(d), the quality degradation of our $L R$ algorithm is almost negligible, i.e., $2.5 \%$ as the number of patterns increases from 10 to 100 . The result again shows our $L R$ algorithm is robust in the number of pattern variations.

\subsection{Evaluating SPASS Runtime}

We now evaluate the efficiency and scalability of the SPASS Runtime compared to the most prominent approach, i.e., SASE [42] Using the SASE approach each pattern in the workload maintains its own event data-structures. The metric used in these experiments is the average execution time, i.e., the total pattern execution times (see Section 5.1) over all window slides divided by the number of window slides. It shows how quickly our SPASS Runtime produces the results for all pattern queries. That is the main concern from the application users' point of view. We vary three key parameters: (1) the length of shared sub-patterns, (2) the number of sequence patterns sharing the same sub-pattern, and (3) the window size. We work with three different workloads over the stock stream [1]. Workload $W 1$ consists of only $S E Q$ patterns, $W 2$ has mixed $S E Q$ and $A N D$ patterns, while $W 3$ has $S E Q$ patterns with ! sub-patterns.

In addition, we also evaluate the effectiveness of the SPASS weight assignment, concurrency mechanism, and overhead of statistics collection. In Sec. 5.4, we further compare our SPASS system against existing sub-pattern selection strategies such as prefixsharing [14], suffix-sharing and random sub-pattern selection.

Effect of varying the window size $w$. As demonstrated in Figure 12(a), the window size $w$ of the workloads is varied from $500 \mathrm{~ms}$ to $2000 \mathrm{~ms}$. The three workloads have 20 patterns each. The average execution time is computed for each window slide. As $w$ increases, each window contains a larger number of events to process. This leads to more sharing opportunities and hence greater performance benefits. Figure 12(a) demonstrates that as the window size increases, the average execution time of both shared and unshared executions increases. The shared execution strategy avoids repeated computations. For both data sets, the execution time without sharing is approximately 16 times that of our SPASS execution when the window size is $500 \mathrm{~ms}$. Moreover, as the window size increases from $500 \mathrm{~ms}$ to $2000 \mathrm{~ms}$ the performance of the unshared approach degrades significantly for all 3 workloads, while the SPASS shared execution exhibits a robust trend. At $w=2000 \mathrm{~ms}$, the unshared approach is approximately 26 times slower than our SPASS shared approach.

Effect of varying the number of shared patterns $k$. In this experiments, we vary the number of patterns in each workload $k$ from 10 to 100 . Here the length of the patterns (i.e., the number of event types in the patterns) is kept unchanged. As the number of shared patterns increases, we observe that our SPASS Runtime is 20 times faster compared to the unshared approach on average (Figure 12(b)). This difference in average execution time becomes 


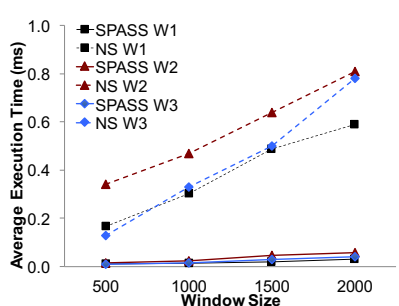

(a) Varying Window Size $(w)$

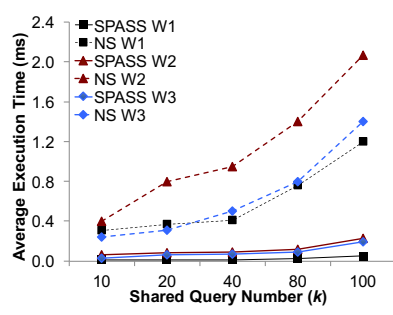

(b) Varying Pattern Number $(k)$

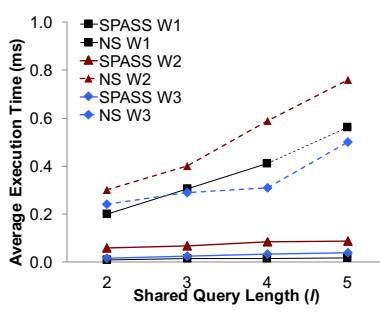

(c) Varying Pattern Length $(l)$

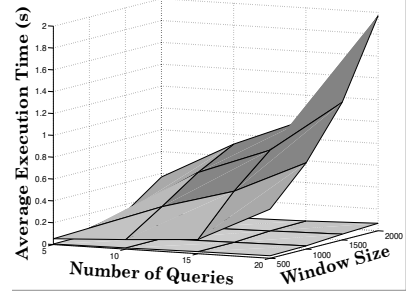

(d) Varying All Together

Figure 12: SPASS Shared Execution with Varying Parameters

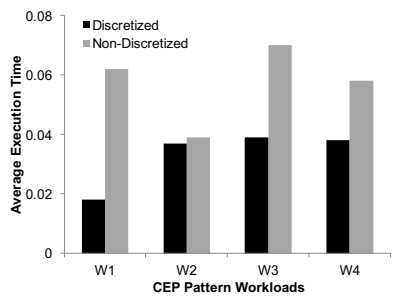

(a) Weight Accuracy

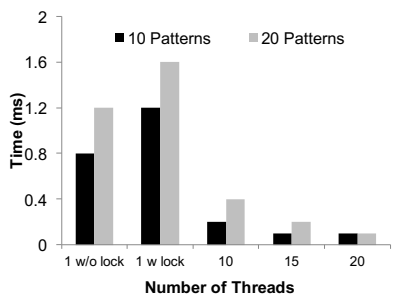

(b) Concurrency

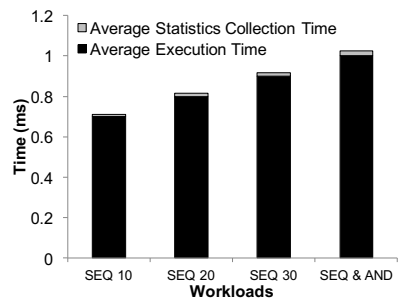

(c) Statistics Overhead

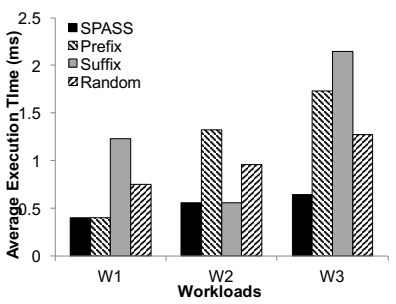

(d) Comparison with SOA

Figure 13: SPASS Runtime Performance

more significant when the number of patterns sharing common subpatterns increases. This is because our shared execution avoids repeated computations by exploiting the shared views.

Effect of varying the shared sub-pattern length $l$. In this experiment we vary the length of the shared sub-patterns in each workload from 2 to 5 . The window size is fixed at $1000 \mathrm{~ms}$ for each workload. As the shared common sub-pattern length is increased, the repeated computations are saved to a greater extent. The average execution time shown in Figure 12(c) confirms the efficiency of the SPASS shared execution. As the length of the sequence patterns increases, the average execution times of both approaches increase. However, by sharing the computations of the shared sub-patterns, the average execution time is reduced by an average factor of 25 times from the unshared approach.

Effect of varying the window size $w$ and the number of patterns $k$. Having seen the effects of one parameter at a time, we now vary two key parameters together to evaluate their interplay. We obtain a surface chart comparing the performance of shared and unshared execution strategies. Figure 12(d) shows the window sizes and number of pattern queries on the two planar ( $\mathrm{X}$ and $\mathrm{Y}$ ) axes. The vertical $(Z)$ axis shows the average execution time. The surface for the shared approach is much closer to the XY plane while the unshared approach diverges from the shared plane as either window size or the pattern numbers or both are increased. As the window size and the number of patterns sharing a sub-pattern both increase, the shared execution achieves more performance gains. On average the shared approach performs 17 times faster average execution time compared to the unshared approach.

Evaluating the effectiveness of SPASS weight assignment. We now illustrate the effectiveness of our weight assignment approach to sub-patterns by analyzing redundancies in the sub-pattern match computation at a fine versus at a coarse granularity. We consider 4 different workloads of 10 pattern queries each. For each workload, the patterns are first run independently to obtain the exact counts of sub-pattern matches as described in Sec. 3.1. Then the time is discretized into fine $1 \mathrm{sec}$ intervals and the weights are computed. This results in a sharing plan $S P_{D}$. The alternate solution is to assign weight to the sub-patterns without discretizing time with the resulting sharing plan called $S P_{N D}$. The average execution time for each sharing plan is shown in Figure 13(a). On average, $S P_{D}$ performs 2.5 times faster than $S P_{N D}$ confirming the effectiveness of the discretized weight assignment approach.

Evaluating SPASS concurrency. In this experiment, we illustrate the efficiency of our proposed sequence transaction model with S2PL protocol. We use two workloads with 10 and 20 patterns each. Figure 13(b) shows that the average execution time with varying the number of threads. The first pair of bars represents the execution time of patterns run as a shared single threaded system without any locking mechanism. The second pair of bars corresponds to the execution time of the two workloads run with a single thread with the locking mechanism turned on. The difference between these two pairs of bars shows the overhead of adding the locking algorithm. Finally the following 3 pairs of bars show the execution time of running the two workloads with 10, 15 and 20 threads. As expected the performance improves with an increasing number of threads. However after adding a certain number of threads, 15 in this case, there is no further improvements in execution time.

Overhead of statistics collection in SPASS Optimizer. In this experiment, we have evaluated the runtime overhead of statistics collection for SPASS weight assignment relative to the overall query execution cost. As depicted in Figure 13(c), the overhead is negligible across all scenarios. On average $1.2 \%$ of the average execution time is spent on statistics collection for workloads composed of $S E Q$ queries. The reason is threefold. First, the statistics are collected periodically as described in Sec 3.1. Second, we exploit the lightweight counting scheme of pattern matches [33] that is pushed into the sequence construction process. Third, our SPASS system only collects statistics for the common sub-patterns rather than all possible sub-patterns. For a mixed workload of $S E Q$ and 
$A N D$ queries, the overhead of the statistics collection is slightly higher, around $2 \%$ of the overall execution time. This is because the SPASS Runtime has to collect statistics for different permutations of the $A N D$ queries.

\subsection{SPASS versus State-of-the-Art Sub-Pattern Selection Strategies}

In this experiment (Figure 13(d)), we compare the SPASS Optimizer sub-pattern selection against existing sub-pattern selection strategies such as prefix-sharing [14], suffix-sharing and random sub-pattern selection. We use three different workloads with 20 queries each to compare the average execution time for the entire workload using each of these approaches. The workload $W 1$ is characterized by 4 sets of 5 patterns sharing common prefixes across the queries. These patterns do not have any other sub-patterns in common. In this case both prefix-sharing and SPASS produce the same pattern sharing plan. They naturally outperform the other strategies in the order of random sub-pattern selection followed last by suffix-sharing. Similarly, the workload $W 2$ consists of 4 sets of patterns with common suffixes. Again our SPASS Optimizer performs as good as suffix-sharing. In the third workload $W 3$, the queries have common sub-patterns that not necessarily are a prefix or suffix sub-pattern. As expected, the SPASS system again outperforms all three state-of-the-art strategies at least 2 fold.

\section{RELATED WORK}

Complex Event Processing. Many CEP systems [6, 3, 42] have been proposed to process sequence pattern queries over event streams. SASE [3, 42] and Cayuga [6] follow an NFA-based approach for detecting sequence patterns where the arrival of events into the system are marked by state transitions. As opposed to NFA-based event processing, ZStream [29] uses tree-based query plans for both the logical and physical representation of query patterns. While these alternate operator implementations could also be used in the SPASS optimizer by adjusting the cost model, it is not the focus of this work. However, none of those systems treat the multiple pattern sharing problem as a first-class citizen.

There have also been some work on stream transaction model [39, 7]. While the model in [7] is general enough to support a broad range of stream queries, it does not take the append only characteristics of event streams into account. In [39], another transaction modelwas proposed for supporting concurrent updates in an active event processing system. Our transaction model in the context of sequence pattern sharing is significantly different from both of the above. Also our locking protocols are customized specifically for shared sequence execution exploiting multi-core architectures.

Multiple Query Optimization. Multiple query optimization (MQO) has been well studied $[9,11,18]$ in static databases. However, optimizing multiple sequence pattern queries introduces new dimensions to the existing MQO problem due to time-ordered event correlations within and across patterns. Hence techniques for tackling the MQO problem in relational databases such as materialized views [11] and common sub-expression sharing $[9,18]$ are not sufficient for sharing sequence patterns over event streams.

MQO has received attention in the context of continuous query processing perspective too. The data stream processing system TelegraphCQ [10] has shared query processing as part of its native architecture. In [28], an adaptive tuple-level sharing technique was proposed to share multiple query processing over data streams with fluctuations. However, routing individual tuples between operators adds significant overheads. Our work instead produces a stable sharing plan [25] and re-optimizes only if there is significant change in statistics.
Preliminary efforts of merging the NFAs representing multiple event queries were made in RUMOR [23] based on the Cayuga [6] system. Their rule-based optimization follows a set of pre-defined steps that merge common sub-expressions across queries. A costbased approach to decide which sub-expressions to share has not been provided. Further, they do not support the concurrent execution of multiple queries with shared sub-expressions. The XMLfiltering work also adopts NFA-based processing [13, 14]. While merging NFAs has been explored in this context [13], it is limited to prefix-matching. In contrast, SPASS supports sharing of subpatterns at any position within a sequence pattern, i.e., it is not necessarily limited to a prefix or a suffix only. In [22], the authors also incorporate materialization based optimization to improve the performance of processing a large number of XML stream queries. However, their approach takes neither window constraints nor event correlations into consideration, which are key factors in our SPASS optimization. Further they do not provide any techniques for maintaining views nor concurrently accessing them by multiple queries.

Materialized View Selection. The idea of using views to improve the query processing performance has been studied in static databases [4, 15, 30, 37]. The prior work primarily focussed on minimizing the overall query execution time by selecting a set of views to materialize, given limited memory space. Mistry et al. [30] proposed a greedy algorithm for selecting a set of views without any guarantees of optimality. Shukla et al. [37] targeted optimal view selection for aggregation queries but not for general sequence pattern queries. Our SPASS system tackles the more general sequence pattern sharing problem with time-ordered event correlations while still guaranteeing an optimality bound.

OctopusDB [15] addresses the problem of designing a good layout for different data access patterns without knowing the exact query patterns up front through adaptive view storage. However, its techniques are designed to optimize the access of different storage structures, rather than sharing common computations among different workloads. DBToaster [4] exploits viewlet transforms, a recursive finite differencing technique, to materialize a query and a set of deltas as views for incremental maintenance. They do not focus on windowed queries and hence fail to tackle the problem of time-ordered event correlations within and across patterns.

In the context of complex event processing, the continuous sliding views mechanism was proposed in [34] to address the problem of view maintenance over event streams for a single query. In our work, we now extend this mechanism with optimization techniques to support shared continuous sliding views for multiple windowed sequence pattern queries. Our proposed techniques provide on concurrent access to shared views for reading, for updating, and for view purging in a timely fashion for memory efficiency without missing or duplicating results.

\section{CONCLUSION}

This work addresses the sequence pattern sharing problem on event streams using the SPASS technology. The proposed SPASS Optimizer leverages event correlations within and across patterns to find an effective sharing plan. We proposed an approximate polynomial-time algorithm to find a shared pattern plan covering all sequence patterns with a guaranteed optimality bound. The SPASS Runtime then exploits the shared continuous sliding view technology to execute this shared pattern plan. A sequence transaction model on shared views is introduced to define the correctness of concurrent pattern executions. Our sequence-aware locking protocol guarantees correct execution while minimizing delays. SPASS achieves 16 folds performance improvement in CPU utilization compared to state-of-the-art techniques. 


\section{REFERENCES}

[1] I. inetats. stock trade traces. http://davis.wpi.edu/dsrg/stockData/eventstream3.txt.

[2] Stanford Linear Road Benchmark. http://infolab.stanford.edu/stream/cql-benchmark.html.

[3] J. Agrawal, Y. Diao, D. Gyllstrom, and N. Immerman. Efficient pattern matching over event streams. In SIGMOD, pages 147-160, 2008.

[4] Y. Ahmad, O. Kennedy, et al. Dbtoaster: Higher-order delta processing for dynamic, frequently fresh views. $P V L D B$, 5(10):968-979, June 2012.

[5] M. Akdere, U. Çetintemel, and N. Tatbul. Plan-based complex event detection across distributed sources. PVLDB, 1(1):66-77, 2008.

[6] Alan J. Demers and Johannes Gehrke and Biswanath Panda and Mirek Riedewald and others. Cayuga: A general purpose event monitoring system. In CIDR, pages 412-422, 2007.

[7] I. Botan, P. M. Fischer, et al. Transactional stream processing. In EDBT, pages 204-215, 2012.

[8] B. Cadonna, J. Gamper, and M. H. Bohlen. Sequenced event set pattern matching. In EDBT, pages 33-44, 2011.

[9] U. S. Chakravarthy and J. Minker. Multiple query processing in deductive databases using query graphs. In $V L D B$, pages 384-391, 1986.

[10] S. Chandrasekaran, O. Cooper, A. Deshpande, et al. Telegraphcq: Continuous dataflow processing for an uncertain world. In CIDR, 2003.

[11] S. Chaudhuri, R. Krishnamurthy, S. Potamianos, and K. Shim. Optimizing queries with materialized views. In ICDE, pages 190 - 200, 1995.

[12] J. Chen, D. J. DeWitt, et al. Niagaracq: A scalable continuous query system for internet databases. In $A C M$ SIGMOD Record, pages 379-390, 2000.

[13] Y. Diao, M. Altinel, M. J. Franklin, H. Zhang, and P. Fischer. Path sharing and predicate evaluation for high-performance xml filtering. ACM TODS, 28(4):467-516, 2003.

[14] Y. Diao, P. Fischer, M. J. Franklin, and R. To. Yfilter: Efficient and scalable filtering of xml documents. In ICDE, pages 341-342, 2002.

[15] J. Dittrich and A. Jindal. Towards a one size fits all database architecture. In CIDR, pages 195-198, 2011.

[16] M. G. Elfeky, W. G. Aref, et al. Stagger: Periodicity mining of data streams using expanding sliding windows. In ICDM, pages 188-199, 2006.

[17] G. Giannikis, G. Alonso, and D. Kossmann. Shareddb: killing one thousand queries with one stone. PVLDB, 5(6):526-537, 2012.

[18] G. Giannikis, P. Unterbrunner, et al. Crescando. In SIGMOD, pages 1227-1230, 2010.

[19] J. Grant and J. Minker. On optimizing the evaluation of a set of expressions. International Journal of Computer \& Information Sciences, pages 179-191, 1982.

[20] J. M. Hellerstein and M. Stonebraker. Predicate migration: Optimizing queries with expensive predicates. In SIGMOD, pages 267-276, 1993.

[21] D. Hermelin, D. Rawitz, et al. The minimum substring cover problem. In International Conference on Approximation and Online Algorithms, pages 170-183, 2008.

[22] M. Hong, A. J. Demers, et al. Massively multi-query join processing in publish/subscribe systems. In SIGMOD, pages 761-772, 2007.
[23] M. Hong, M. Riedewald, C. Koch, J. Gehrke, and A. Demers. Rule-based multi-query optimization. In $E D B T$, pages 120-131, 2009.

[24] S. Krishnamurthy, M. J. Franklin, et al. The case for precision sharing. In $V L D B$, pages 972-984, 2004.

[25] C. Lei, E. Rundensteiner, and et al. Robust distributed stream processing. In ICDE, pages 817-828, 2013.

[26] M. Liu, E. Rundensteiner, and et al. High-performance nested cep query processing over event streams. In ICDE, pages 123 - 134, April, 2011.

[27] M. Liu, E. Rundensteiner, and et.al. E-cube: Multi-dimensional event sequence analysis using hierarchical pattern query sharing. In SIGMOD, pages 889-900, 2011.

[28] S. Madden and et al. Continuously adaptive continuous queries over streams. In SIGMOD, pages 49-60, 2002.

[29] Y. Mei and S. Madden. Zstream: a cost-based query processor for adaptively detecting composite events. In SIGMOD, pages 193-206, 2009.

[30] H. Mistry, P. Roy, S. Sudarshan, and K. Ramamritham. Materialized view selection and maintenance using multi-query optimization. In ACM SIGMOD Record, volume 30, pages 307-318, 2001.

[31] R. V. Nehme, K. Works, C. Lei, E. A. Rundensteiner, and E. Bertino. Multi-route query processing and optimization. Journal of Computer and System Sciences, 79(3):312-329, 2013.

[32] N. Onose, A. Deutsch, Y. Papakonstantinou, and E. Curtmola. Rewriting nested xml queries using nested views. In SIGMOD, pages 443-454, 2006.

[33] Y. Qi, L. Cao, et al. Complex event analytics: online aggregation of stream sequence patterns. In SIGMOD, pages 229-240, 2014.

[34] M. Ray, E. Rundensteiner, et al. High-performance complex event processing using continuous sliding views. In $E D B T$, pages 525-536, 2013.

[35] P. Roy and et al. Efficient and extensible algorithms for multi query optimization. In SIGMOD, pages 249-260, 2000.

[36] N. P. Schultz-Møller et al. Distributed complex event processing with query rewriting. In $D E B S$, pages $4: 1-4: 12$, 2009.

[37] A. Shukla, P. Deshpande, J. F. Naughton, et al. Materialized view selection for multidimensional datasets. In $V L D B$, volume 98, pages 488-499, 1998.

[38] A. Thomasian. Two-phase locking performance and its thrashing behavior. ACM TODS, 18(4):579-625, 1993.

[39] D. Wang, E. A. Rundensteiner, and R. T. Ellison. Active complex event processing over event streams. PVLDB, 4(10):634-645, 2011.

[40] S. Wang, M. Hao, et al. Chaos: A data stream analysis architecture for enterprise applications. In 2009 IEEE conference on commerce and enterprise computing, pages 33-40, 2009.

[41] S. Wang, E. Rundensteiner, et al. State-slice: New paradigm of multi-query optimization of window-based stream queries. In $V L D B$, pages 619-630, 2006.

[42] E. Wu, Y. Diao, et al. High-performance complex event processing over streams. In SIGMOD, pages 407-418, 2006.

[43] Y. Zhu, E. A. Rundensteiner, and G. T. Heineman. Dynamic plan migration for continuous queries over data streams. In SIGMOD, 2004. 


\section{APPENDIX}

\section{A. SPASS OPTIMIZER}

\section{A.1 Event Pattern Cost Model}

Cost of Single $S E Q$ Pattern Execution. For a sequence pattern operator $P_{i}=S E Q\left(E_{1}, \ldots, E_{n}\right)$, the computation costs $C_{P i}$ of $P_{i}$ using NFA-based pattern evaluation are formulated in Equation 5. Essentially it is the product of the cardinality of the event type $E_{i}$ and $E_{j}$ with the intra-event correlation that gives an estimate of the number of $E_{j}$ events would follow $E_{i}$ events in a given time window. The automata-based execution technique provides a special indexing technique [42] that can find sequence pattern matches without doing a cross-product of all events arriving in a given window. The total computation cost for $k$ different query patterns would be $\sum_{i=1}^{k} C_{P_{i}}$.

$$
C_{P_{i}}=\sum_{i=1}^{n}\left|E_{i}\right| *\left[\prod_{j=0}^{i}\left|E_{j}\right| * C_{\text {Intra }}\left(E_{j}, E_{j+1}\right) * P_{E_{i}}\right]
$$

Cost of Single $A N D$ Pattern Execution. For an $A N D$ pattern operator $P_{i}=A N D\left(E_{1}, \ldots, E_{n}\right)$, the computation costs $C_{P i}$ of $P_{i}$ using a evaluation are formulated in Equation 6. This cost is based on the execution model discussed in [8].

$$
C_{P_{i}}=\prod_{i=1}^{n}\left|E_{i}\right| * P_{E_{i}}
$$

Cost of Shared Pattern Execution. The shared execution of pattern queries chops the query into smaller sub-patterns $\left[S P_{1} \ldots S P_{k}\right]$. Each sub-pattern $S P_{i}$ is computed once and only once with the respective cost $C_{S P_{i}}$ and shared across patterns. Several sub-patterns are then re-combined to form final results for pattern queries in the workload.

Assume two patterns $P_{1}$ and $P_{2}$ that share a common sub-pattern $S P_{12} . \quad P_{1}$ is composed of sub-pattern $S P_{1}$ and $S P_{12}$ while $P_{2}$ is composed of $S P_{2}$ and $S P_{12}$. The inter-query correlation factor gives reflects how many of these sub-pattern matches are common across the two parent patterns. Thus the shared cost of execution is obtained by subtracting the product of execution cost of the common sub-pattern with the the inter-query correlation from the sum cost of executing the parent patterns individually. This cost is shown in Equation 7. It is straightforward to generalize it for multiple sub-patterns shared across multiple queries.

$$
C_{S}\left(P_{1}, P_{2}\right)=C_{P_{1}}+C_{P_{2}}-C_{\text {Inter }}\left(P_{1}, P_{2}, S P_{12}\right) * C_{S P 12}
$$

\section{A.2 Multiple Windows and Predicates in SPASS Optimization}

Sequence patterns in a workload might have different windows as well as predicates specified. The handling of queries with different predicates and window sizes has been already addressed by previous research using sliced time windows and shared data fragments [41]. In this paper, the SPASS optimizer's cost model takes such window and predicate specifications into account. However, its main focus is to identify shared sub-patterns based on event correlations within or across multiple pattern queries.

Our cost model captures redundant sub-pattern computations. Consider the two sub-patterns $S P_{1}=S E Q(A$ (A.color=orange \& A.color=blue $), B)$ and $S P_{2}=S E Q(A(A . c o l o r=r e d \&$ A.color $=b l u e), B)$. If most patterns satisfy the predicate $(A$.color $=b l u e)$, then there could be a high degree of redundancy.
Similarly pattern queries with different sized window parameters may still share sub-pattern matches across each other in our SPASS optimizer. Our redundancy ratio implicitly captures this information during the shared pattern plan generation. The reason is that the sample time period used to analyze sub-pattern matches is guaranteed to be larger than the largest window size among all pattern queries sharing this sub-pattern. For instance consider patterns $P_{1}=S E Q(A, B, C)$ with window $=10$ and $P_{2}=S E Q(A, B, D)$ with window $=100$. The events forming the pattern matches for $P_{1}$ can be directly reused by $P_{2}$.

\section{A.3 Sharing Across Nested Patterns}

In this work we focus primarily on optimizing flat CEP queries. However, SPASS system supports sharing operators across nested event patterns without considering optimization. Given a pattern $\operatorname{SEQ}(X, A N D(A, B, C), Y)$ and another pattern $A N D(A, B, C)$, it identifies the two $A N D$ operators with the same operands. It then shares the computation of this sub-pattern across two queries.

There has been prior work on rewriting nested patterns to exploit sharing opportunities $[32,26]$. These techniques could be exploited to rewrite nested CEP patterns before applying our optimization principle. However using these principles [26] we often end up with an exponential number of flat queries which might result in exponential blow up of the input to the MSC problem. For instance the query $\operatorname{SEQ}(X, A N D(A, B, C), Y$,$) when rewritten, would result in$ 6 different $S E Q$ queries that each need to be run.

\section{A.4 Proof of Lemma 1}

Proof. Sub-pattern Sharing $\subseteq$ MSC Problem. Given a set of pattern queries $W$, each pattern $P_{i}$ is given as $S E Q\left(E_{1}, E_{2}, \ldots\right.$, $\left.E_{n}\right)$. For each event type we assign a specific letter. Thus each pattern is translated to a string of letters ' $E_{1} E_{2} \ldots E_{n}$ '. Thus, the pattern query workload $W$ is analogous to a set of strings $S$. The set of common sub-patterns across any two or more pattern queries is equivalent to substrings common across two or more strings. The weight function of $R R$ (defined in Eq. 4) is $\Theta(W) \rightarrow \mathbb{Q}_{0,1}$ as $R R$ is a ratio of two natural numbers where the denominator is guaranteed to be greater than the numerator. We define a cover $C_{W}$ of $W$ to be a set of sub-patterns in $\Theta(W)$, where for each $P_{i} \in$ $W$ there exist $S P_{1}, \ldots, S P_{k} \in C_{W}$, with $P_{i}=\left(S P_{1} \cdot \ldots S P_{k}\right)$. Thus finding the optimal solution for the MSC problem is equivalent to finding a cover $C_{W}$ of the pattern workload $W$ composed of a set of sub-patterns that has a minimum total $R R$, namely $\operatorname{Min}\left(R R\left(C_{W}\right)\right)$ for all possible covers $C_{W}$ of $W$.

MSC $\subseteq$ Sub-pattern Sharing Problem. It is straightforward to reduce the MSC problem to the sub-pattern sharing problem as well.

\section{B. SPASS RUNTIME}

\section{B.1 Single Sequence Execution using Continuous Sliding Views}

Continuous sliding views are effectively cached intermediate results for sequence sub-patterns. We briefly enumerate the steps in for a single sequence pattern execution using continuous views. We assume here that a sequence pattern $S$ is composed of two subpatterns $S P_{1}$ and $S P_{2}$ such that $S P_{1}$ is the right sub-pattern and $S P_{2}$ is the left sub-pattern. Sub-pattern matches for $S P_{2}$ are maintained in a view view $w_{S P 1}$.

1. When an event instance $e_{i}$ arrives at time $i$, it is inserted into its respective storage unit called event stack. 
2. Events in the event stacks that have timestamps $<i$ - window are purged from the stacks.

3. If $e_{i}$ is a triggering event then the right-most sub-pattern $S P_{1}$ is triggered execution. Sequence matches are computed from the events that are currently in the event stacks within the current window.

4. Once pattern matches for $S P_{1}$ have been computed, $S P_{2}$ is then executed following a right-to-left order processing. Matches for sub-pattern $S P_{2}$ are then re-used from view $w_{S 1}$. Continuous sliding views are augmented with a view indicator that indicates the completeness status of the view. If the view is found to contain all required pattern matches, then they are re-used.

5. If the view ${ }_{S P 1}$ is found to be incomplete, new pattern matches for $S P_{2}$ are computed from the event stacks and appended to the view. The view indicator is also updated to indicate the updated state of the view. At the same time old pattern matches are purged from the view.

EXAMPLE 7. Consider the pattern $\operatorname{SEQ}(A, B, C, D)$ is executed as a combination of two sub-patterns $S P_{c}=S E Q(A, B)$ and $S P_{1}=S E Q(C, D)$, the matches for sub-pattern $S P_{c}$ are cached in the continuous view view ${ }_{S P c}$ (Figure 14(a)). Pattern $S P_{1}$ is executed first followed by $S P_{c}$. When the triggering event $d_{18}$ arrives, the sub-pattern $S P_{1}$ triggers pattern matching and the new match $\left\{\left\langle c_{16} d_{18}\right\rangle\right\}$ is computed. The timestamp of the left most event in sub-pattern $S P_{1}$ constrains the pattern match for $S P_{c}$. Thus for the match $\left\{\left\langle c_{16} d_{18}\right\rangle\right\}$, the timestamp of the leftmost event $C$ is 16 . The matches for $S P_{c}$ that can form complete matches for $P_{1}$ are those that occur within the time-interval of [ $t_{\text {current }}$ - window, $\left.t_{\text {leftevent }}\right]=[18-10,16]$. Hence for this triggering event, $S P_{c}$ is only computed for the range $[8,16]$. Sliding views are augmented with special indicators called rightBound that indicates if the view contains all pattern matches up to a certain time. In this case the view has already contained sub-pattern matches for $S E Q(A, B)$ up to timestamp 16 . Thus the sub-pattern matches are directly reused from the view. When event instance $d_{21}$ arrives to the system (Figure 14(b)), the event $a_{11}$ expires as the window $=$ 10. Consequently all sub-pattern matches containing $a_{11}$ are also purged from the view. Further, $d_{21}$ forms new sequence matches for the sub-pattern $S E Q(A, B)=\left\{\left\langle c_{16} d_{11}\right\rangle,\left\langle c_{20} d_{21}\right\rangle\right\}$. Now the subpattern $S E Q(A, B)$ is looked up for the interval [11, 20]. However, the view currently contains sub-pattern matches up to a timestamp of 16 . Thus the view requires an update thus adding the new subpattern match $\left\{\left\langle a_{12} b_{19}\right\rangle\right\}$ to the view. Finally the indicator of the view is updated to 20.

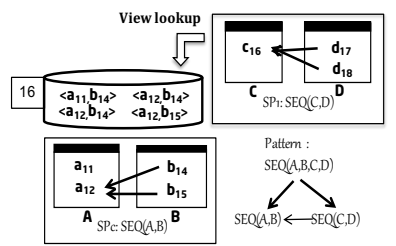

(a) Read

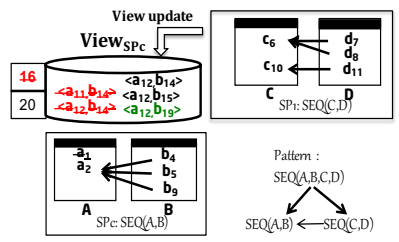

(b) Update
Figure 14: Single Sequence Execution using Continuous Views

\section{B.2 Multiple Windows and Predicates in SPASS Runtime}

Support for Different Windows. Multiple sequence patterns could have different window sizes. The shared continuous sliding view technology could support multiple sequence patterns with different window sizes by maintaining pattern matches in the view for the larger window. The reuse, append, and purge operations can be easily modified to support this feature.

window $_{\max }$. In order to support multiple windows, each shared view is associated with a window $w_{\max }$. For the set of patterns $\left\{P_{1}, \ldots P_{k}\right\}$ that access a view $V i e w_{S P i}$ each pattern $P_{i}$ has an associated window window $_{i}$. Then the largest window among all these window $_{i}$ is designated as window max. View $w_{S P i}$ contains all sub-pattern matches of $S P_{i}$ that satisfy this largest window window $w_{\max }$.

Modified View Reuse, Append, and Purge. During the reuse phase, each pattern $P_{i}$ thus filters out only those pattern matches that satisfy window $_{i}$ constraint. During the append phase pattern matches for the sub-pattern $S P_{i}$ must be computed while satisfying the window $_{\max }$ constraint. The purge operation on the view must be performed only when sub-pattern matches are to be expired out of the largest window, i.e., sub-pattern matches containing event instances $e_{i}$ such that $i<t_{\text {current }}-$ window $_{\max }$.

EXAMPLE 8. For example, in Figure 7, if $P_{2}$ had a window with size 20 and $P_{1}$ had a window size 10 , then the view $w_{S P}$ could contain pattern matches such as $\left\langle a_{11}, b_{1} 3\right\rangle$ where b.ts a.ts $>10$. Such matches would not qualify for pattern $P_{1}$. These matches must be filtered out by $P_{1}$ during the reuse phase. Further, if newly arrived events $c_{25}$ and $d_{30}$ formed a pattern match \langle $\left.c_{25}, d_{30}\right\rangle$ for the sub-pattern $S P_{1}$ the view would be updated for the interval $\left[t_{\text {current }}-\right.$ window $\left._{P 1}, 25\right]=[20,25]$. However if the view $w_{S c}$ is not complete, the view update must be done for an interval [ $t_{\text {current }}-$ window $\left.w_{\max }, 25\right]=[10,25]$.

Support for Different Predicates. Next we extend our above treatment by considering how the presence of predicates in the pattern queries influence the shared execution process. Sequence patterns could have selection predicates specified on events or join predicates across events such as $P_{1}=S E Q(A$ :A.color $=$ blue, $B: B . i d=A . i d)$. In SPASS Runtime, we exploit the approaches on predicate sharing that have been extensively studied in the literature [20]. If a sub-pattern is shared across two patterns yet the two patterns specify different selection predicate on them, the sub-pattern is rewritten to satisfy both selection predicates using existing query rewriting techniques [27]. For instance, consider the above pattern $P_{1}$ and pattern $P_{2}=S E Q(A:$ A.color $=$ black, $B: B . i d=A . i d)$. In this case the selection predicates A.color $=$ blue and A.color $=$ black on $P_{1}$ and $P_{2}$ could be rewritten to $($ A.color $=$ black or A.color $=$ black $)$. For equijoin-like predicates across the entire sequence pattern (e.g., $P_{1}$ ), the views could be partitioned by the join attribute value [34]. Thus predicate migration techniques such as push-down and pull-up [20] which are effectively orthogonal to shared continuous sliding views can be adopted in our SPASS execution.

\section{B.3 Lock Compatibility}

Lock Compatibility on Event Buffers. The read and append locks on buffers are completely independent of each other. Similarly, two appends could take place at the same time on a buffer. This is due to the assumption that duplicate events do not arrive in the event stream. Thus the actions of appending two events to the same buffer are independent. Similarly, if a transaction is appending to the buffer, while another transaction has requested a read lock on the buffer, it means the buffer must already contain the events up to the timestamp requested by the read lock. This is guaranteed by the sequential lock assignment phase. 


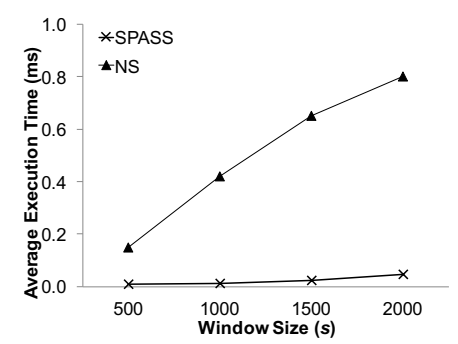

(a) Varying Window Size $(w)$

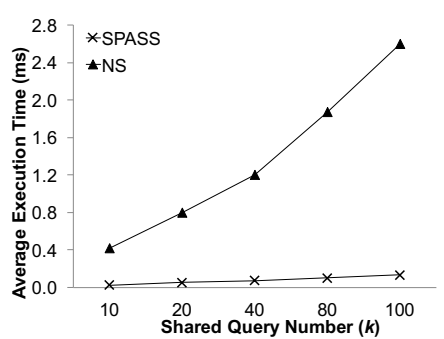

(b) Varying Pattern Number $(k)$

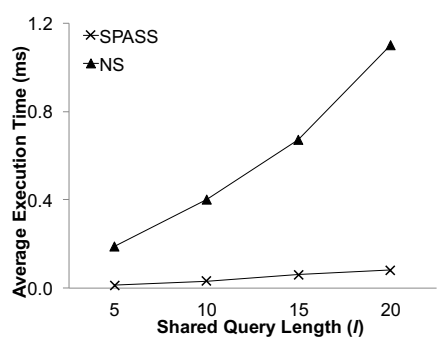

(c) Varying Pattern Length $(l)$

Figure 15: Shared Execution with Varying Parameters (Linear Road)

Lock Compatibility on Shared Views. In terms of transactions on views, if an $A_{V i e w}$ operation is in progress, another $R_{V i e w}$ transaction should be allowed to execute due to the sequential lock assignment phase. The sequential lock assignment must predetermine that a transaction only requests a read lock on a view if the validity interval of the view at the time of arrival of this event indicates that the view already contains the required pattern matches. Otherwise it must create two locks namely $R_{b u f f e r(s)}$ and $A_{V i e w}$. Two append operations are not allowed on a view at the same time. This avoids the creation of duplicate results.

Purge Lock. Finally the $P_{B u f f e r}$ and $P_{V i e w}$ locks are considered most restrictive. Notice that purge locks could be denied even when there simply exists another request for a lock on that resource, even if it has not yet been granted. That is in order to grant a purge request, the transaction manager must make sure that the lock buffer for that resource does not contain any read requests on that resource that may try to read events or pattern matches purged by this purge transaction. This prevents any missing pattern matches.

\section{EXPERIMENTAL EVALUATION}

In this set of experiments, we evaluate the efficiency and scal- ability of our SPASS system with another dataset from the Linear Road Benchmark [2]. The dataset consists of car locations with timestamps. The pattern query workload comprises of finding sequences of locations that a car has traveled with a period of time. We vary the three parameters used in Sec. 5.3: (1) the length of shared sub-patterns, (2) the number of sequence patterns sharing the same sub-pattern, and (3) the window size. As depicted in Figure 15(a), as the window size is increased from $500 \mathrm{~s}$ to $2000 \mathrm{~s}$, the advantage of our SPASS system compared to the unshared solution in the average execution time increases from 10 folds to 20 folds. The reason is that the number of event tuples sharable in each window is substantially increased in the larger window. Similarly, when the number of the queries is increased from 10 to 100 as shown in Figure 15(b), the winning margin of our solution over unshared solution significantly increases, as more computational savings are obtained by exploiting more sharing opportunities in a larger query workload. Lastly, in Figure 15(b), when the length of queries is increased, the average execution time of our SPASS system is relatively stable compared to the unshared approach as expected. This is because the longer the common sub-patterns are, the more they contribute to the computational savings obtained by the queries sharing them. 